\title{
An overview on the phase diagram of the frustrated two-leg ladder model
}

\author{
T. Hakobyan日, J.H. Hetherington $\mathbb{\theta}$ and M. Roger \\ Service de Physique de l'Etat Condensé, Commissariat à l'Energie Atomique, \\ Centre d'Etudes de Saclay, 91191 Gif sur Yvette Cedex, France.
}

(November 4, 2018)

\begin{abstract}
Using Density-Matrix Renormalization Group, we investigate the general phase diagram of the frustrated two-leg ladder with Heisenberg interactions along legs, rungs and diagonals. We confirm that all antiferromagnetic gapped states belong to the same universality class as the Haldane phase. In a three-dimensional phase-diagram, we determine a continuous surface with singularities in the string-order parameter or its first derivative, corresponding to a transition between two Haldane phases with different topological order. Some parts of this transition surface are critical with zero gap and vanishing string-order parameter. In the complementary parts, the transition is first order with finite gap and string order. The boundary of this surface with the ferromagnetic region is a critical end line, when the surface is critical and a triple line anywhere else. Part of this boundary coincides with the exactly soluble model proposed by D. V. Dmitriev, V. Ya Krivnov and A. A. Ovchinnikov [Phys. Rev. B 56, 5985 (1997)].
\end{abstract}

$75.10 \mathrm{Jm}$

\section{INTRODUCTION}

$\mathcal{N}$-legged spin ladders are formed by assembling $\mathcal{N}$ spin- $\frac{1}{2}$ chains one next to the other. Many weaklycoupled ladder systems have now been synthetized. Among them, the family $\mathrm{Sr}_{n-1} \mathrm{Cu}_{n+1} \mathrm{O}_{2 n}$ consists in weakly coupled $\frac{1}{2}(n+1)$-legged ladders which are obtained from the $\mathrm{CuO}_{2}$ planes of the parent compound $\mathrm{SrCuO}_{2}$. After the suggestion that mechanisms similar to those occurring in the $\mathrm{CuO}_{2}$ planes of cuprate ceramics may also lead to superconductivityl, an intense theoretical and experimental activity has been developed on these systems. The doped spin-ladder compound $\mathrm{Sr}_{14-x} \mathrm{Ca}_{x} \mathrm{Cu}_{24} \mathrm{O}_{41}$ is superconducting up to $10 \mathrm{~K}$ under pressure. Some theoretical tools which are specific to onedimensional systems make the theoretical understanding of ladders easier than that of the $\mathrm{CuO}_{2}$ planes responsible for the high-temperature superconductivity in cuprate ceramics. It is believed that the mechanisms leading to superconductivity are similar in both systems and the understanding of spin ladders should give some insight in the physics of the more complex two-dimensional superconducting systems.

A first step in the theoretical understanding of these systems is the study of undoped ladders in which the spin interactions are of Heisenberg type. The exact solution of the spin- $\frac{1}{2}$ Heisenberg chain has been known for seventy years from the Bethe ansatz2. The quantum fluctuations prevent long-range antiferromagnetic order, there is no gap in the excitation spectrum and spin-spin correlations decay in power law as a function of the distance. However, the crossover from ladders to the square lattice, by increasing the number of coupled chains, is far from being smooth. While ladders with an odd number of legs exhibit properties similar to those of a single chain, i.e. gapless excitations and a power-law fall-off of spin-spin correlations, ladders with an even number of legs have a finite energy gap to the lowest spin-1 excitation and exponential decay of spin-spin correlations. These remarkable quantum properties are remininiscent of those, first conjectured by Haldane 3 , concerning the spin- $\frac{n}{2}$ Heisenberg chain with, respectively, $\mathrm{n}$ odd and $\mathrm{n}$ even. Therefore, a natural question arises: how are the ladder phases related to the phases in the spin chain?

(a)

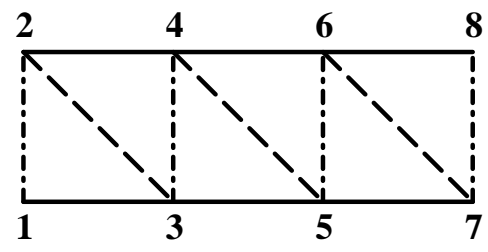

(b)

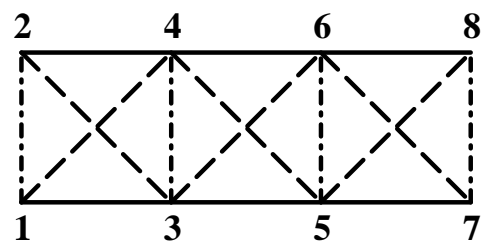

(c)

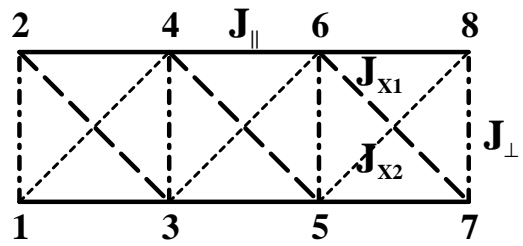

FIG. 1. Frustrated-ladder models: (a) The "Zig-Zag chain". (b) Frustrated ladder with equal diagonal interactions. (c) The more general model studied in this paper 
For the two-leg ladder, this question has been partly answered by Whitet. He considered a two-leg ladder with additional interaction along one diagonal of each plaquette [Fig. 1 (a)] and showed by varying this interaction that there is a continuous path from the Haldane to the ladder phase which does not cross any phase boundary or critical points. Some controversial arguments were published recently by Wang $\mathrm{E}$. He considered a ladder with additional equal interactions along both diagonals of each plaquette [Fig. 1 (b)]. Using Density-Matrix Renormalization Group (DMRG) calculations 6 he proved the existence of a first-order transition line and suggested the occurrence of a phase-transition from the Haldane-phase to a gapped singlet phase with different sensitivity with respect to boundary conditions.

It is therefore interesting to study the general "frustrated two-leg ladder model" [Fig. 1 (c)], including two different diagonal interactions in the plaquettes, which contains both previous works as particular cases and to draw a general phase diagram. We thus consider the following Hamiltonian:

$$
\begin{aligned}
& H=2 \sum_{i=1}^{L}\left[\quad J_{\|}\left(\mathbf{S}_{\mathbf{i}} \cdot \mathbf{S}_{\mathbf{i}+\mathbf{2}}+\mathbf{S}_{\mathbf{2} \mathbf{i}-\mathbf{1}} \cdot \mathbf{S}_{\mathbf{2} \mathbf{i}+\mathbf{1}}\right)+\right. \\
& J_{\perp} \mathbf{S}_{\mathbf{2} \mathbf{i}-\mathbf{1}} \cdot \mathbf{S}_{\mathbf{2} \mathbf{i}}+ \\
& \left.J_{X 1} \mathbf{S}_{\mathbf{2} \mathbf{i}} \cdot \mathbf{S}_{2 \mathbf{i}+\mathbf{1}}+J_{X 2} \mathbf{S}_{2 \mathbf{i}-\mathbf{1}} \cdot \mathbf{S}_{\mathbf{2}+\mathbf{2}}\right]
\end{aligned}
$$

( $L$ is the ladder length and the number of spins is $N=2 L$ ) and propose a general three-dimensional phase diagram for this model. There is an obvious symmetry of this Hamiltonian by exchanging $J_{X 1}$ and $J_{X 2}$ and it is more convenient to use:

$$
\begin{aligned}
& \mathcal{S}=J_{X 1}+J_{X 2} \quad \text { and } \\
& \mathcal{D}=J_{X 1}-J_{X 2}
\end{aligned}
$$

as parameters. We choose the following energy scaling:

$$
\left|2 J_{\|}\right|+\left|J_{\perp}\right|+|\mathcal{S}|+|\mathcal{D}|=1
$$

Due to the symmetry, it is sufficient to consider $\mathcal{D}>0$. We choose as independent variables $x=2 J_{\|}, y=\mathcal{S}$, $z=J_{\perp}$ and $\mathcal{D}$ is given by the previous relation. The variables $\{x, y, z\}$ obey: $|x|+|y|+|z|<1$ and are thus contained in the regular octahedron represented in Fig. 2.

Using Density-Matrix Renormalization Group (DMRG), Conformal Field Theory and some exact analytical results, we draw transition surfaces separating various phases and identify their critical parts. We review the most important results obtained earlier for particular cases, including the spin-1 and spin- $\frac{1}{2}$ Heisenberg chain, the frustrated spin- 1 and spin- $\frac{1}{2}$ chains with interactions between nearest and next nearest neighbors, the dimerized chain, and the usual ladder, which are all gathered in this general phase diagram. We do not put any restriction on the sign of the four exchange parameters and provide a new insight in the regions with negative (ferromagnetic) interactions.
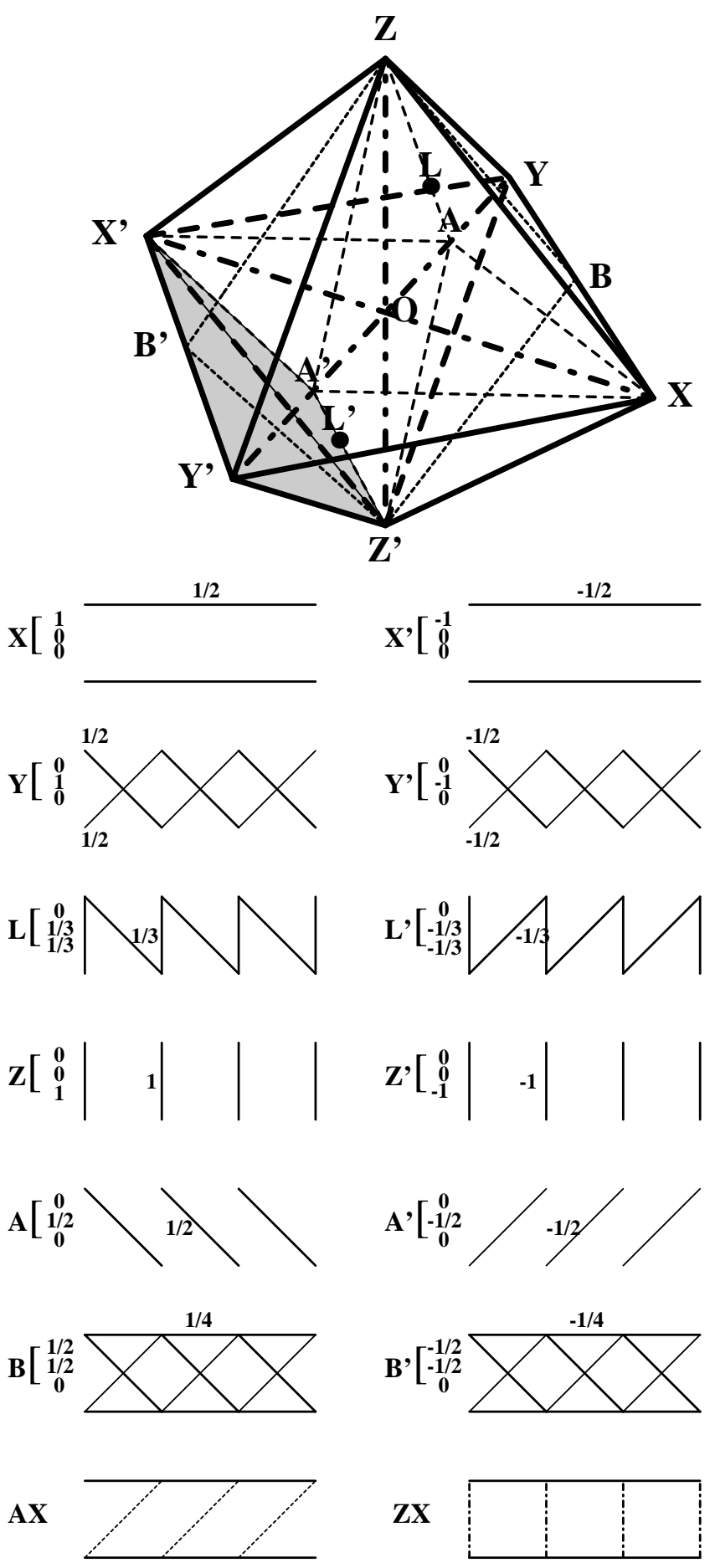

FIG. 2. The regular octahedron inscribed in a sphere of radius 1 , representing the parameter-space of our Hamiltonian [Eq. (1)] with the energy scaling of Eq. (3). Some remarkable points with their $\{x, y, z\}$ coordinates are listed: the spin- $\frac{1}{2}$ Heisenberg antiferromagnetic $(X, Y, L)$ or ferromagnetic ( $\left.\mathrm{X}^{\prime}, \mathrm{Y}^{\prime}, \mathrm{L}^{\prime}\right)$ chain, products of independent dimers $(\mathrm{A}, \mathrm{Z})$ or triplet pairs $\left(\mathrm{A}^{\prime}, \mathrm{Z}^{\prime}\right)$, the usual ladder (AX and ZX segments) etc... The "Zig-Zag" chain represented in Fig. 1 (a) corresponds to the surface of the irregular octahedron ( $\left.\mathrm{AA}^{\prime}, \mathrm{XX}, \mathrm{ZZ}^{\prime}\right)$. In the gray volume, all interactions are negative (ferromagnetic). 
We begin with the study of the vicinity of particular points of this octahedron where the low-lying eigenstates can be mapped onto those of the Haldane spin-1 or spin- $\frac{1}{2}$ chain (section II), and introduce three different phases: two topologically distinct gapped states and the ferromagnetic phase. In our three-dimensional phase diagram, the points $\mathrm{X}, \mathrm{Y}, \mathrm{L}$ representing the spin- $\frac{1}{2}$ antiferromagnetic Heisenberg chain appear as particular critical points lying in zero-gap critical surfaces separating the two gapped Haldane phases. In section III, we briefly describe the method that we use to determine transition surfaces between different phases: a Density-Matrix Renormalization group algorithm, with "suitable" open boundary conditions.

Section IV is devoted to particular planes in the octahedron corresponding the the ladder model represented in Fig. 1 (a). This model can also be viewed as a "ZigZag" Heisenberg chain with nearest and next-nearest neighbor interactions and has already been extensively studied. We briefly review the most important results obtained for this particular case. Using Lanczös diagonalisation and DMRG, we investigate in section $\mathrm{V}$ the whole phase diagram in the octahedron and draw transition surfaces between the three different phases identified in section II. We show that no other phase appears.

\section{IDENTIFICATION OF DIFFERENT PHASES OF THE PHASE-DIAGRAM}

\section{A. Evidence for two topologically distinct spin-1 Haldane phases}

The points Z' (all interactions null, except $J_{\perp}=-1$ ) and A' (all interactions null except $J_{X 2}=-1 / 2$ ) represent independent pairs of spins $1 / 2$ coupled ferromagnetically along rungs and along plaquette diagonals respectively. The $3^{L}$ degenerate ground-state is identical to that of $L$ independent spins-1: $\tilde{\mathbf{S}}_{\mathbf{i}}=\left(\mathbf{S}_{\mathbf{2} \mathbf{i}}+\mathbf{S}_{\mathbf{2 i - 1}}\right)$ at Z' and $\tilde{\mathbf{S}}_{\mathbf{i}}=\left(\mathbf{S}_{\mathbf{2} \mathbf{i}}+\mathbf{S}_{\mathbf{2} \mathbf{i}-\mathbf{3}}\right)$ at $\mathrm{A}^{\prime}$. In the neighborhood of both points, degenerate perturbation theory is relevant as far as the other coupling parameters are much smaller than the gap between the $3^{L}$ degenerate ground-state and the first excited state (i.e. $<<1$ ). The split $3^{L}$ lowest levels can be mapped onto the states of a chain of $\mathrm{L}$ interacting spins-1 with effective Hamiltonian (at first order in the coupling parameters):

$$
H_{e f f}=\frac{2 J_{\|}+J_{X 1}+J_{X 2}}{2} \sum_{i=1}^{L} \tilde{\mathbf{S}}_{\mathbf{i}} \cdot \tilde{\mathbf{S}}_{\mathbf{i}+\mathbf{1}}
$$

in the neighborhood of point Z' and

$$
H_{e f f}=\frac{2 J_{\|}+J_{\perp}}{2} \sum_{i=1}^{L} \tilde{\mathbf{S}}_{\mathbf{i}} \cdot \tilde{\mathbf{S}}_{\mathbf{i}+\mathbf{1}}+\frac{J_{X 1}}{2} \sum_{i=1}^{L} \tilde{\mathbf{S}}_{\mathbf{i}} \cdot \tilde{\mathbf{S}}_{\mathbf{i}+\mathbf{2}}
$$

in the neighborhood of point $\mathrm{A}^{\prime}$
The Hamiltonian (4) for $\left(2 J_{\|}+J_{X 1}+J_{X 2}\right)>0$ and the Hamiltonian (5) for $2 J_{\|}>0$ and $J_{X 1}=0\left(J_{X 1}=0\right.$ is satisfied in the planes $\left.\mathrm{A}^{\prime} \mathrm{XZ}, \mathrm{A}^{\prime} \mathrm{XZ} \mathrm{X}^{\prime}, \mathrm{A}^{\prime} \mathrm{X}^{\prime} \mathrm{Z}\right)$ represent the spin-1 Haldane chain. For $J_{X 1}=0$ both spin-1 Hamiltonians (4) and (5) are formally identical. However, they represent different topological configurations of the original ladder: the spin- 1 operators $\tilde{\mathbf{S}}_{\mathbf{i}}$ represent the projection on the triplet state of the sum of a pair of spins- $\frac{1}{2}$ along one rung in Hamiltonian (4) and along one diagonal of a plaquette in Hamiltonian (5).

The most important breakthrough for the understanding of the antiferromagnetic spin-1 Heisenberg chain has been the discovery by Affleck-Kennedy-Lieb-Tasaki (AKLT) of an exactly soluble Hamiltonian differing from the Heisenberg model only by addition to the Hamiltonian of a biquadratic term:

$$
H_{A K L T}=\sum_{i}\left[\tilde{\mathbf{S}}_{\mathbf{i}} \cdot \tilde{\mathbf{S}}_{\mathbf{i}+\mathbf{1}}+\frac{1}{3}\left(\tilde{\mathbf{S}}_{\mathbf{i}} \cdot \tilde{\mathbf{S}}_{\mathbf{i}+\mathbf{1}}\right)^{2}\right]
$$

Its exact ground-state called "Valence Bond Solid" (VBS) is constructed out of valence bonds, it is nondegenerate and breaks no symmetry. It can be represented as schematized in Fig. 3 (a).

- The spin-1 variables are expressed as a sum of two spin- $\frac{1}{2}$ variables

- Let $\psi_{\alpha, \beta}$ denote a spin-1 state in terms of the symmetrized spin- $\frac{1}{2}$ variables:

$$
\begin{array}{lc}
\psi_{++}= & \mid++>, \\
\psi_{+-}=\psi_{-+}=(|+->+|-+>) / \sqrt{2} \\
\psi_{--}= & \mid-->.
\end{array}
$$

- Each spin- $\frac{1}{2}$ is contracted with one spin- $\frac{1}{2}$ at a neighbor site into a singlet state: a "valence bond". Contraction of two spin- $\frac{1}{2}$ variables into a singlet state can be written as $\epsilon^{\alpha \beta} \mid \alpha \beta>/ \sqrt{2}$, where $\epsilon^{\alpha, \beta}$ represents the antisymmetric tensor: $\epsilon^{++}=\epsilon^{--}=$ 0 and $\epsilon^{+-}=-\epsilon^{-+}=1$

- The contractions are such that every spin-1 is connected to both its neighbors by a valence bond and the VBS state can be written:

$$
\begin{array}{r}
\mid \Psi_{V B S}>=2^{-N / 2} \psi_{\alpha_{1} \beta_{1}} \epsilon^{\beta_{1} \alpha_{2}} \psi_{\alpha_{2} \beta_{2}} \epsilon^{\beta_{2} \alpha_{3}} \ldots \\
\psi_{\alpha_{i} \beta_{i}} \epsilon^{\beta_{i} \alpha_{i+1}} \cdots \psi_{\alpha_{L} \beta_{L}} \epsilon^{\beta_{L} \alpha_{1}}
\end{array}
$$

The VBS state, used as variational state for the Heisenberg spin-1 chain, gives an upper bound of the energy which is only a few percent higher than the best approximates through DMRG and is thought to capture the essentials of the physics of the this model. The VBS state has some "hidden" topological long-range orderl. 
(a)

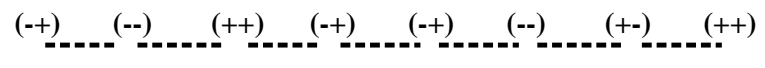

(b)

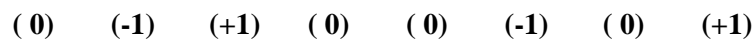

FIG. 3. Schematic view of a typical component of the VBS state described by Eq. 8. (a) The dashed links represent Valence-Bonds between two fictive neighboring spins- $\frac{1}{2}$. (b) Sequence of spin-1 $S^{z}$ values corresponding to (a): the non-zero spins are in a Néel order $(-1,1,-1,1, \ldots$.

It is easy to see that each term of the sum in the previous relation has $S_{i}^{z}$ sequences ordered in a way shown schematically in Fig. 3 (b). If we discard the sites with $S_{i}^{z}=0$, the remaining $S_{i}^{z}= \pm 1$ sites have Néel order: $|1,-1,1,-1,1,-1, \cdots\rangle$. This topological order is characterized by the non-local "string-order parameter":

$$
\mathcal{O}^{z}(|m-l|)=\left\langle\tilde{S}_{l}^{z} \exp \left(i \pi \sum_{k=l+1}^{k=m-1} \tilde{S}_{k}^{z}\right) \tilde{S}_{m}^{z}\right\rangle
$$

A normalized string-order parameter:

$$
\tilde{\mathcal{O}}^{z}(d)=\frac{-\mathcal{O}^{z}(d)}{<\left(\tilde{S}^{z}\right)^{2}>^{2}}
$$

has been later introduced by Whited. Its value for $d \rightarrow \infty$ is 1 for the VBS state and 0.84 for the Heisenberg spin- 1 chain.

From the previous discussion about the neighborhoods of points Z' and A' in the phase diagram, it is natural to introduce two string-order parameters:

- $\mathcal{O}_{\perp}(d)$ obtained with $\tilde{S}_{i}^{z}=\left(S_{2 i}^{z}+S_{2 i-1}^{z}\right)$ in Eq. 9, with triplet pairs of spins along rungs

- $\mathcal{O}_{X}(d)$ obtained with $\tilde{S}_{i}^{z}=\left(S_{2 i}^{z}+S_{2 i-3}^{z}\right)$ in Eq. 9, with triplet pairs of spins along one diagonal of each plaquette

These two topologically distinct Haldane phases, that we now call $H D_{\perp}$ and $H D_{X}$, characterized respectively by the string-order parameters $\mathcal{O}_{\perp}$ and $\mathcal{O}_{\perp}$ have been introduced in a recent work by Kim et al.10, with slightly different notations. They are schematized in Fig. 4. Using field-theoretical methods, Kim et al. have found evidences for some transition lines between these two Haldane phases around some particular points of the phase diagram corresponding to two-decoupled Heisenberg chains (see sections IV and V).

With $J_{X 1}$ strictly positive, the full Hamiltonian (5) represents the "frustrated" antiferromagnetic Heisenberg $\mathrm{S}=1$ chain, with interactions $J_{1}=\left(2 J_{\|}+J_{\perp}\right) / 4$ and $J_{2}=J_{X 1} / 4$ between first and second neighbors. This model has been studied by Kolezhuk et al.11 They find a sharp discontinuity in the string-order parameter $\mathcal{O}_{X}$ at $J_{2} / J_{1} \approx 0.7444$, suggesting a first-order transition. The significance of this first-order transition will appear in section $\mathrm{V}$, when placed in the framework of the general phase-diagram.

\section{B. The antiferromagnetic spin-1/2 Heisenberg chain}

In Fig. 2a, the points $X:\left(2 J_{\|}=1\right.$, all other $J$ 's null $)$ and $Y:\left(J_{X 1}=J_{X 2}=1 / 2\right.$, all other $J$ 's null $)$ represent two decoupled spin- $\frac{1}{2}$ antiferromagnetic Heisenberg chains, and the point $L\left(J_{\perp}=J_{X 1}=1 / 3\right.$, all other $J$ 's null) represents a single spin- $\frac{1}{2}$ antiferromagnetic Heisenberg chain. The exact solution of this Hamiltonian is known from the Bethe Ansatz2. There is no gap in the excitation spectrum and the spin-spin correlations decrease as power law in terms of the distance. In sections IV and $\mathrm{V}$, we shall prove that these points lie in critical surfaces separating the two Haldane phases with different topological order.

\section{The ferromagnetic state}

In the grey volume A'Y'X'Z' of the octahedron [Fig. 2 (a)], all interactions are negative. The groundstate is ferromagnetic. It has gapless spin-wave excitations. The ferromagnetic state obviously extends beyond this volume. Its limits will be determined in sections IV and V.

(a)

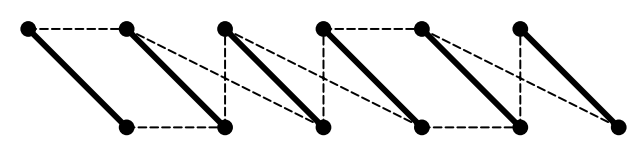

(b)

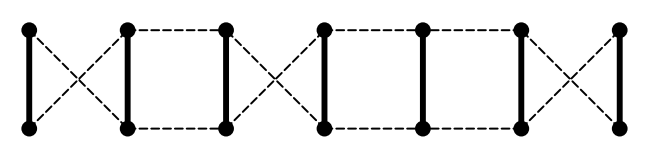

FIG. 4. The two topologically distinct Haldane phases $H D_{X}$ (a) and $H D_{\perp}$ (b). The heavy links represent triplet pairing and the dashed links schematize Valence Bonds. 
(a)
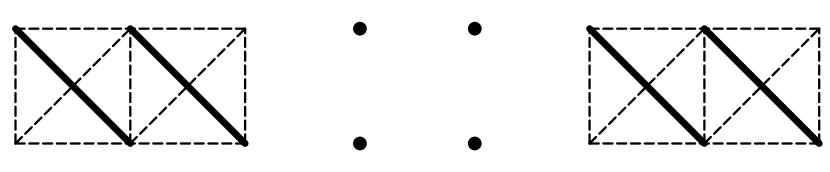

(b)
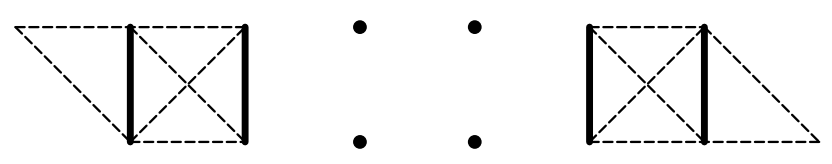

FIG. 5. Growing algorithms used in DMRG calculation with free boundaries: (a) in the $H D_{X}$ Haldane Phase, where triplet pairing occurs along diagonals, (b) in the $H D_{\perp} \mathrm{Hal}-$ dane phase, where triplet pairing occurs along rungs. In either case, the ground state is non degenerate.

\section{DMRG WITH SUITABLE BOUNDARY CONDITIONS}

Our purpose is to determine transition lines or surfaces separating different phases, in particular the two topologically distinct Haldane phases $H D_{\perp}$ and $H D_{X}$ defined in section II. We use the Density-Matrix Renormalization Group (DMRG) method with open boundary conditions which gives much more accurate results than with periodic boundary conditiond $\mathrm{d}$. However, a wellknown inconvenience for the Haldane spin-1 chain with open boundary conditions is the four-fold degeneracy of the ground-state. This degeneracy is easily understood through the description in Fig. 3 (a) and Eq. (8) of the VBS state, which is thought to capture the essential of the physics of the Haldane chain. In Fig. 3 (a), with open boundary conditions, there are two spin- $\frac{1}{2}$ at each end of the chain which are not contracted through a valence bond to any other half-spin. The interaction between these extra half-spins fall off exponentially with $\mathrm{L}$ and in the long-chain limit, there is a four-fold degeneracy of the ground-state due to these two free spins.

In their DMRG treatment of the spin-1 chain, White and Huse remove this inconvenient degeneracy by adding at each end an extra spin- $\frac{1}{2}$ which forms a singlet with half of the last spin-1 in the chain ${ }^{2}$. When applying DMRG to the frustrated ladder problem, the adaptation of this trick is straightforward and already built in the ladder topology. In the $H D_{X}$ phase, we use the boundary conditions shown in Fig. 5 (a) which are generally chosen in treating the ladder problem 5 . In the $H D_{\perp}$ phase, we use other boundary conditions schematized in
Fig. 5 (b). If the ground state is viewed as a VBS state, in either case Figs. 5 (a) and (b) show that there is no "free spin" ("free" means not linked to any another spin with a "valence bond") at either end. Consequently, the ground-state is non degenerate in the long chain limit. In either case our DMRG algorithm for growing the chain length proceeds by adding one rung to each of the right and left Blocks as shown in Fig. 5. What differs is the shape of the initial Block: it is a rectangle in case (a) for the $H D_{X}$ phase and a trapezoïd in case (b) for the $H D_{\perp}$ phase.

Using the same algorithm for any point of the phasediagram (most previous BMRG studies on ladders have proceeded in such a way generally leads to numerical difficulties when crossing the phase-boundary. Suppose that we start from a point in the $H D_{X}$ phase with the suitable algorithm [Fig. 5 (a)] and follow a path to the $H D_{\perp}$ phase, at the transition to the $H D_{\perp}$ phase, the ground-state, which was non-degenerate becomes fourfold degenerate and we need to keep a much larger number of states to obtain a reasonable approximation of the transition point. To determine numerically many transition points in a three-dimensional phase diagram, we need a "cheap" algorithm for calculating a transition point with a reasonable accuracy in a minimum of computer time.

Our algorithm uses the fact that at a phase transition, there are some singularities in the first or second derivatives of the energy. There is a discontinuity in the first derivative if the transition is first order. Here, if the transition is second order the second derivative is generally infinite at the critical point. We thus proceed as follows:

- We choose a path $P_{0} P_{0}^{\prime}$ going from the $H D_{X}$ phase $\left(P_{0}\right)$ to the $H D_{\perp}$ phase $\left(P_{0}^{\prime}\right)$ and parametrized by the variable $u$. Using the algorithm schematized in Fig. 5 (a), we calculate at $P_{0}$ the energy and its first and second derivatives. The first derivative of the energy, with respect to $x=2 J_{\|}, y=\mathcal{S}$ and $z=J_{\perp}$ is obtained by calculating the correlations:

$$
\begin{aligned}
C_{\|}= & <1+4 \mathbf{S}_{\mathbf{i}} \cdot \mathbf{S}_{\mathbf{i}+\mathbf{2}}>/ 2 \\
C_{\perp}= & <1+4 \mathbf{S}_{\mathbf{2 i}-\mathbf{1}} \cdot \mathbf{S}_{\mathbf{2 i}}>/ 2 \\
C_{X 1}= & <1+4 \mathbf{S}_{\mathbf{2 i}} \cdot \mathbf{S}_{\mathbf{2 i + 1}}>/ 2 \\
C_{X 2}= & <1+4 \mathbf{S}_{\mathbf{2} \mathbf{i}-\mathbf{1}} \cdot \mathbf{S}_{\mathbf{2 i + 2}}>/ 2
\end{aligned}
$$

The derivative of the energy $\partial E_{0} / \partial u$ along the path is obtained from the preceding correlations. The second derivative $\partial^{2} E_{0} / \partial u^{2}$ is obtained numerically through the same calculation with an infinitesimal variation on $u$ (chosen at the square root of the numerical precision). An approximate second order polynomial expression of the energy near $P_{0}$ is deduced:

$$
E=E_{0}+s \partial E_{0} / \partial s+\left(s^{2} / 2\right) \partial^{2} E_{0} / \partial s^{2}
$$


- Using the other algorithm schematized in Fig. 5 (b), a polynomial approximation of the energy around $P_{0}^{\prime}$ is obtained in the same way.

- A first approximation $P_{0}^{C}$ of the critical point is given by the intersection of both polynomials.

- A new starting point $P_{1}$ is chosen half way between $P_{0}$ and $P_{0}^{C}$, a new end point $P_{1}^{\prime}$ is chosen half way between $P_{0}^{C}$ and $P_{0}^{\prime}$ and the process is reiterated.

An accuracy of 4 to 5 digits is generally obtained for the critical point after 8 to 10 iterations. We keep 30 to 60 states and use chain length $L \approx 50$. Some accuracy tests we be given in section $\mathrm{V}$.

\section{THE "ZIG-ZAG CHAIN"}

The ladder model, with only one diagonal interaction, represented in Fig. 1 (a) can also be viewed as a spin- $\frac{1}{2}$ dimerized next-nearest neighbor Heisenberg chain. Using the scaling from Eq. (3), the two-dimensional parameter space for this model corresponds to the eight faces of the irregular octahedron XX'AA'ZZ' (Fig. 6). There is an obvious symmetry, in this model, obtained by exchanging rung and diagonal interaction. The symmetry line XLX'L'X, corresponding to equal rung and diagonal interactions splits the parameter surface into two pieces with a one to one correspondence. Due to our non-trivial energy scaling [Eq. (3)], this mapping does not correspond however to simple reflections.

The AXZ face, in which all interactions are antiferromagnetic has been studied for many years. On the symmetry line $\mathrm{XL}$, the point $\mathrm{G}$ corresponding to $J_{\perp}=J_{X \downarrow}=2 J_{\|}$has been considered by Majumdar and Ghosh 13 thirty years ago. The ground state is doubly degenerate and corresponds to a product of singlet pairs (or dimers) along rungs or diagonals. Shastry and Sutherland 14 have later proven that the dimer state along the rungs (resp. diagonals) is the exact ground state on the whole line GZ (resp. GA). Note that dimer states have perfect "string order", characterized by the normalized order parameters $\hat{\theta} \tilde{\mathcal{O}}_{X}=1$ on GZ and $\tilde{\mathcal{O}}_{\perp}=1$ on GA, respectively. Following the path AGZ, the string order parameter $\tilde{\mathcal{O}}_{\perp}$ jumps abruptly from 1 to 0 at the Majumdar-Ghosh point $\mathrm{G}$ and $\tilde{\mathcal{O}}_{\perp}$ jumps respectively from 0 tone. The whole symmetry line LX is a transition line 15 17. According to our notations introduced in section II, it corresponds to a phase transition between the two symmetrical Haldane phases $H D_{X}$ and $H D_{\perp}$. Both ends X and L correspond to the spin- $\frac{1}{2}$ Heisenberg chain. The vicinities of these points have been studied through field-theoretical methods $10.17,18$. The transition is first order near $\mathrm{X}$ and second order near L. On XL the nature of the transition changes from first to second order at a tricritical point T. From field-theoretical results, at $\mathrm{T}$ the spectrum develops a gap between the $\mathrm{S}=0$ ground state and the first excited $\mathrm{S}=1$ states and simultaneously the degeneracy between the two lowest $S=0$ and $\mathrm{S}=1$ excited states is split. Using Lanczös diagonalisation on finite systems, Emery et al 17 have extrapolated in the thermodynamic limit the exchange parameter values at which the energy difference between the lowest $\mathrm{S}=0$ and $\mathrm{S}=1$ excited states vanishes and found $\mathrm{T}$ at $J_{\|} / J_{\perp} \approx 0.241$. Using the same method, we have found no evidence for another tricritical point on the symmetry line LX' in the AX'Z face. Hence TLX' is a critical line probably up to $\mathrm{X}^{\prime}$.

In the A'X'Z' face, all interactions are negative and the ground state is ferromagnetic. The ferromagnetic phase extends further in the neighboring faces: A'X'Z, A'XZ' and AX'Z'. In these faces, the line of transition from ferromagnetic to antiferromagnetic state corresponds to the exactly solvable Hamiltonian studied by Dmitriev, Krivnov and Ovchinnikov 19 and hereafter referred as the DKO model Hamiltonian:

$$
\begin{aligned}
H_{D K O}= & -\sum_{i=1}^{L}\left(\mathbf{S}_{\mathbf{2} \mathbf{i}-\mathbf{1}} \cdot \mathbf{S}_{\mathbf{2} \mathbf{i}}-\frac{1}{4}\right) \\
- & (\nu-1) \sum_{i=1}^{L}\left(\mathbf{S}_{\mathbf{2 i}} \cdot \mathbf{S}_{\mathbf{2} \mathbf{i}+\mathbf{1}}-\frac{1}{4}\right) \\
& +\frac{(\nu-1)}{2 \nu} \sum_{i=1}^{L}\left(\mathbf{S}_{\mathbf{i}} \cdot \mathbf{S}_{\mathbf{i}+\mathbf{2}}-\frac{1}{4}\right)
\end{aligned}
$$

The ferromagnetic ground-state of this Hamiltonian is degenerate with a singlet state, which can be written explicitly 19 . With the scaling defined in Eq. (3), we can write the line of transition in the following parametric form:

- A'X'Z face

$$
\begin{aligned}
& x=2 J_{\mid}=(\nu-1) /\left(1+2 \nu-\nu^{2}\right) \\
& y=\mathcal{S}=J_{X 2}=-\nu /\left(1+2 \nu-\nu^{2}\right) \\
& z=J_{\perp}=-\nu(\nu-1) /\left(1+2 \nu-\nu^{2}\right)
\end{aligned}
$$

with $0<\nu<1$

- AX'Z' face

$$
\begin{aligned}
& x=\quad 2 J_{\mid}=(\nu-1) /\left(1+2 \nu-\nu^{2}\right) \\
& y=\mathcal{S}=J_{X 1}=-\nu(\nu-1) /\left(1+2 \nu-\nu^{2}\right) \\
& z=\quad J_{\perp}=-\nu /\left(1+2 \nu-\nu^{2}\right)
\end{aligned}
$$

with $0<\nu<1$

- $\mathrm{A}^{\prime} \mathrm{XZ}$ face

The line XL' corresponding to $J_{X 2}=J_{\perp}$ is a symmetry line. On the upper side of this line the ferromagnetic transition is parametrized by

$$
\begin{aligned}
& x=2 J_{\|}=(\nu-1) /\left(\nu^{2}+2 \nu-1\right) \\
& y=\mathcal{S}=J_{X 2}=-\nu /\left(\nu^{2}+2 \nu-1\right) \\
& z=J_{\perp}=-\nu(\nu-1) /\left(\nu^{2}+2 \nu-1\right)
\end{aligned}
$$

with $1<\nu<2$. On the other side, we have: 

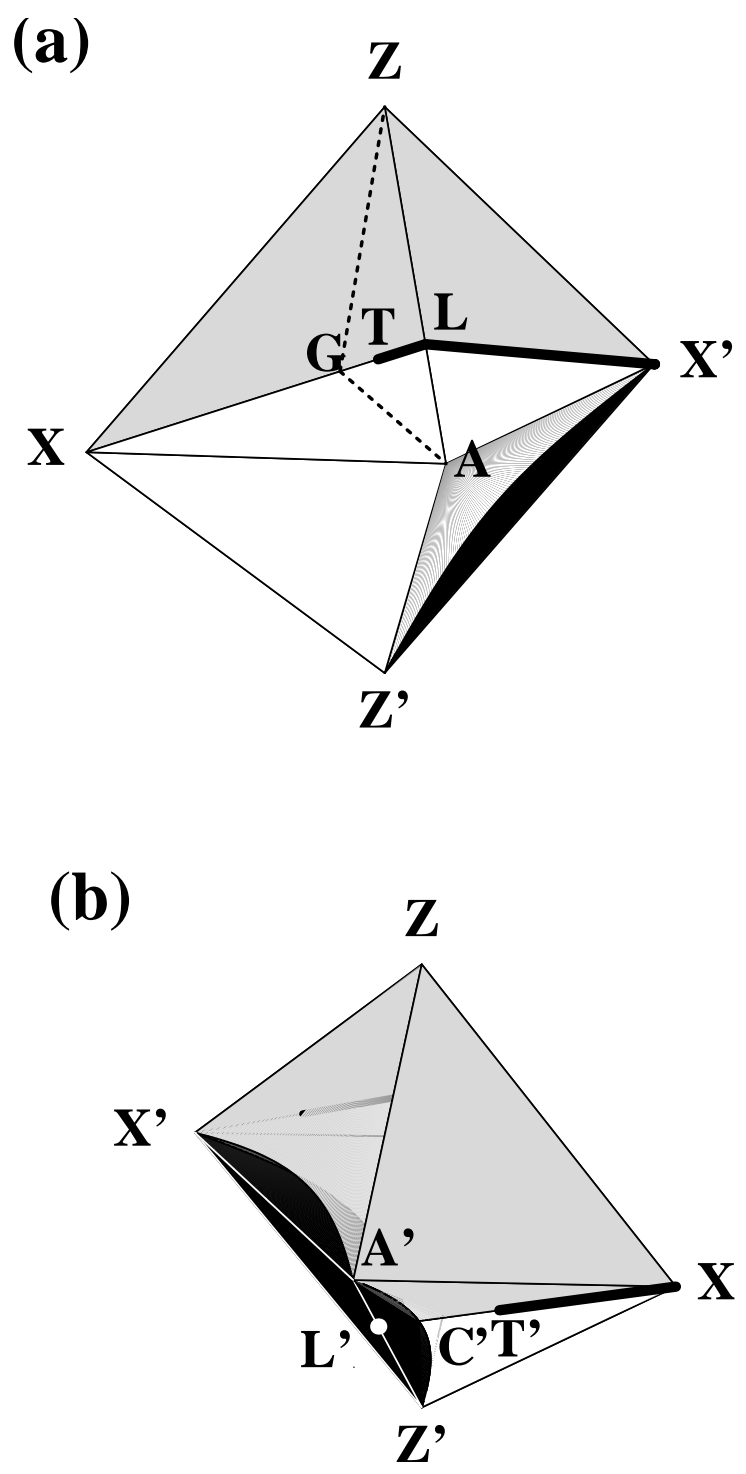

FIG. 6. The surface of the irregular octahedron XX'AA'ZZ' represents the two-dimensional phase diagram of the "Zig-Zag chain" model [Fig. 1 (a)]. The symmetry line XLX'L'X corresponding to equal rung and diagonal interactions splits this surface into two parts which map each other through the exchange of rung and diagonal. Above (resp. below) this line, the antiferromagnetic gray (resp. white) area corresponds to the $H D_{X}$ (resp. $H D_{\perp}$ ) Haldane phase. The black area represents the ferromagnetic state. TLX' and T'X are two zero-gapped critical lines corresponding to second order transition between the two Haldane phases. On TX and T'E', the transition is first order. G represents the Majumdar-Ghosh point. From Shastry and Sutherland, on the thin dotted segments GA and GZ, the ground-state is exact and corresponds to a product of independent dimers

$$
\begin{aligned}
& x=\quad 2 J_{\|}=(\nu-1) /\left(2 \nu^{2}-1\right) \\
& y=\mathcal{S}=J_{X 2}=-\nu(\nu-1) /\left(2 \nu^{2}-1\right) \\
& z=\quad J_{\perp}=-\nu /\left(2 \nu^{2}-1\right)
\end{aligned}
$$

with $1<\nu<2$

In the A'XZ' phase, with ferromagnetic rung and diagonal interactions, the symmetry line XL' meets the ferromagnetic region at C' $(1 / 7,-2 / 7,-2 / 7)$. From fieldtheoretical results $1 \mathrm{~d}$, near $\mathrm{X}$ this line is critical with zero gap and corresponds to a second order phase transition between the two symmetric Haldane phases $H D_{\perp}$ and $H D_{X}$. Using Lanczös diagonalizations on finite systems with $\mathrm{N}$ up to 24 particles and the method previously described 17 we have found that this critical line ends at a tricritical point $\mathrm{T}^{\prime}$ corresponding to $2 J_{\|} \approx 0.42$. From $T$ ' to $C^{\prime}$, the transition between the two symmetrical gapped phases is first order and C' appears as a triple point.

Figure 6 summarizes the results:

- The X'LTXT'C' part of the symmetry line (equal rung and diagonal interactions) corresponds to a phase transition between two symmetric Haldane phases $H D_{\perp}$ and $H D_{X}$ corresponding through the exchange of rung and diagonal.

- X'LT and XT' (heavy segments in Fig. 6) are critical lines with zero gap and second order transition while XT and C'T' corresponds to a first order transition with degenerate ground state and finite gap.

- On either side of the critical line, the $H D_{\perp}$ and $H D_{X}$ phase correspond through a simple symmetry. Consequently, the topology of their groundstates differ, but they have the same thermodynamic properties and same critical exponents near the critical line. In the usual sense of "universality" refering to critical expenents, they belong to the same universality class 20 .

Our study of the general ladder model [Fig. 1 (c)] will now extend this simple diagram in three dimensions. Critical lines will be generalized into critical surface etc...

\section{THE GENERAL PHASE-DIAGRAM}

\section{A. Some exact critical lines and surfaces}

In the preceding section, we have seen that in the surface of the irregular octahedron XX'AA'ZZ' corresponding to the "Zig-Zag" chain the ferromagnetic boundary is exactly known. We shall extend here this exact boundary to a part of the whole ferromagnetic volume. We have put forward the lines GZ and GA where a dimer state is the exact ground state. We shall prove that the exact dimer state extends over some planar surface and 
find exactly the boundary between this surface and the ferromagnetic phase.

Let us rewrite the Hamiltonian [Eq. (1)] as a sum over plaquettes:

$$
\begin{array}{ccc}
H= & \sum_{i=1}^{L} H_{i}^{\square} \quad \text { with } \\
H_{i}^{\square}= & 2 J_{\|}\left(\mathbf{S}_{\mathbf{i}} \cdot \mathbf{S}_{\mathbf{i}+\mathbf{2}}+\mathbf{S}_{\mathbf{2} \mathbf{i}-\mathbf{1}} \cdot \mathbf{S}_{\mathbf{2} \mathbf{i}+\mathbf{1}}\right)+ \\
& J_{\perp} \mathbf{S}_{\mathbf{2} \mathbf{i}-\mathbf{1}} \cdot \mathbf{S}_{\mathbf{2} \mathbf{i}}+ \\
& \left.2 J_{X \mathbf{1}} \mathbf{S}_{\mathbf{2} \mathbf{i}} \cdot \mathbf{S}_{\mathbf{2} \mathbf{i}+\mathbf{1}}+2 J_{X 2} \mathbf{S}_{\mathbf{2} \mathbf{i}-\mathbf{1}} \cdot \mathbf{S}_{\mathbf{2} \mathbf{i}+\mathbf{2}}\right]
\end{array}
$$

The eigenvalues of $H_{i}^{\square}$ for an isolated plaquette are:

$$
\begin{array}{cc}
\lambda_{1}= & -3 J_{\perp} / 2 \\
\lambda_{2}= & J_{\perp} / 2+J_{X 1}+J_{X 2} \\
\lambda_{3}= & J_{\perp} / 2-2\left(J_{X 1}+J_{X 2}\right) \\
\lambda_{4}= & -J_{\perp} / 2 \\
\lambda_{5}= & -\frac{1}{2}\left(J_{X 1}+J_{X 2}+\left[J_{\perp}^{2}+5 J_{X 1}^{2}\right.\right. \\
& \left.\left.-6 J_{X 1} J_{X 2}+5 J_{X 2}^{2}-2 J_{\perp}\left(J_{X 1}+J_{X 2}\right)\right]^{\frac{1}{2}}\right) \\
\lambda_{6}= & -\frac{1}{2}\left(J_{X 1}+J_{X 2}-\left[J_{\perp}^{2}+5 J_{X 1}^{2}\right.\right. \\
& \left.\left.-6 J_{X 1} J_{X 2}+5 J_{X 2}^{2}-2 J_{\perp}\left(J_{X 1}+J_{X 2}\right)\right]^{\frac{1}{2}}\right)
\end{array}
$$

The eigenvalue $\lambda_{1}$ corresponds to a product of rung dimers. It is straightforward to check that it is an exact eigenstate of the total Hamiltonian $H$ if $2 J_{\|}=\mathcal{S}=$ $J_{X 1}+J_{X 2}$. The eigenvalue $\lambda_{2}$ corresponds to the ferromagnetic state, it is always an exact eigenstate of the full Hamiltonian H. A lower bound to the ground-state energy per unit length $E_{0} / L$ of the full Hamiltonian $H$ is given by the lowest eigenvalue $\lambda_{\min }$ of $H_{i}^{\square}$. If the lowest eigenvalue is $\lambda_{2}$, the ground state is ferromagnetic. The volume where $\lambda_{\min }=\lambda_{2}$ provides an inner envelope to the ferromagnetic region. After little algebra, we find that this inner envelope is defined by the surfaces:

$$
w=4 J_{X 1} J_{X 2}+\left(J_{X 1}+J_{X 2}\right)\left(2 J_{\|}+J_{\perp}\right)+2 J_{\|} J_{\perp}=0
$$

inside the irregular tetrahedron XX'AA'ZZ' and

$$
w^{\prime}=\left(J_{X 1}+J_{X 2}\right)\left(2 J_{\|}+J_{\perp}\right)+2 J_{\|} J_{\perp}=0
$$

outside this tetrahedron.

On another hand, an outer envelope of the ferromagnetic region is provided by a simple calculation of spinwaves instabilities in the ferromagnetic phase. For the "Zig-Zag chain", Kolezhuk and Mikeska21 have already pointed out that a simple spin-wave instability calculation in the ferromagnetic region gives exactly the DKO transition line. Introducing two kinds of bosons for representing the spin operators on two different legs, we find one optical and one acoustic mode. The energy of the acoustic mode at $k \rightarrow 0$ is:

$$
E_{s w}^{a c}=-w k^{2}
$$

where $w$ corresponds to the expression given in Eq. (20) The ferromagnetic state is instable with respect to spinwaves for $w>0$. The surface $w=0$ thus provides an outer envelope to the ferromagnetic region. Inside the irregular octahedron XX'AA'ZZ', we have thus provided an outer and an inner envelope which coincide. The surface $w=0$ [Eq. (20)] is thus an exact boundary of the ferromagnetic region.

It is also worth paying some particular attention to the plane ZBZ'B' of the regular octahedron (see Fig. 2) which corresponds to $2 J_{\|}=\left(J_{X 1}+J_{X 2}\right)$. As pointed out above, in that plane, the dimer-state with independent dimers along the rungs is an exact eigenstate of the Hamiltonian.

As previously emphasised this dimer state has perfect string order $\tilde{\mathcal{O}}_{X}=1$ and corresponds to the $H D_{X}$ Haldane phase. Its exact first order transition line with the ferromagnetic phase is given by the equation:

$$
\lambda_{1}=\lambda_{2} \quad \text { i.e. } \quad 2 J_{\perp}+J_{X 1}+J_{X 2}=0
$$

from point $\mathrm{D}=(-2 / 5,-2 / 5,1 / 5)$ to point $C=$ $(-2 / 7,-2 / 7,1 / 7)$ corresponding to DKO model (see Fig. 7). The other part CZ' of the transition line from the ferromagnetic to the $H D_{\perp}$ phase is given exactly by Eq. (20).

The line ZBZ' with $J_{X 1}=J_{X 2}=J_{\|}>0$ corresponds to the so called "composite-spin model" 22. The Hamiltonian can be written as:

$$
\begin{aligned}
H & =H_{1}+H_{2} \quad \text { with : } \\
H_{1} & =2 J_{\|} \sum_{i=1}^{L} \tilde{\mathbf{S}}_{\mathbf{i}} \cdot \tilde{\mathbf{S}}_{\mathbf{i}+\mathbf{1}} \\
H_{2} & =2 J_{\perp} \sum_{i=1}^{L} \mathbf{S}_{\mathbf{2 i}-\mathbf{1}} \cdot \mathbf{S}_{\mathbf{2} \mathbf{i}}
\end{aligned}
$$

where $\tilde{\mathbf{S}}_{\mathbf{i}}=\mathbf{S}_{\mathbf{2} \mathbf{i}-\mathbf{1}}+\mathbf{S}_{\mathbf{2} \mathbf{i}}$ represents the sum of a pair of spins- $1 / 2$ on a rung. Hamiltonians $H_{1}$ and $H_{2}$ commute. The ground state $E_{2}^{0}$ of $H_{2}$ corresponds to a product of dimers along rungs i.e: $E_{2}^{0} / L=-3 J_{\perp} / 2$. It is easy to check that it is also an eigenstate of $H$ with eigenvalue $E_{2}^{0}$. The states with all pairs $\tilde{\mathbf{S}}_{\mathbf{i}}=\mathbf{S}_{\mathbf{2} \mathbf{i}-\mathbf{1}}+\mathbf{S}_{\mathbf{2} \mathbf{i}}$ along the rungs in a triplet state are degenerate eigenstates of $\mathrm{H}_{2}$ with eigenvalues $E_{2}=L J_{\perp} / 2$. The subspace spanned by these $3^{L}$ states corresponds to the spin- 1 chain. The low-energy spectrum of $H_{1}$ corresponds to that of the spin-1 Haldane chain, and the eigenstates of $H_{1}$ are also eigenstates of $H$. The ground state of the spin-1 Haldane chain has an energy $E_{1}^{0} \approx-1.401484039 \times 2 J_{\|}$from DMRG calculations 12 and is an eigenstate of $H$ with energy $E_{1}=E_{1}^{0}+E_{2}$. Starting from the dimer phase at point $\mathrm{Z}$, we have a first order transition to the Haldane phase $H D_{\perp}$ at the crossing between these two energy levels $E_{1}^{0}+E_{2}=E_{2}^{0}$ corresponding to a critical value $J_{\perp} / J_{\|} \approx 1.401484039$ (point $\mathrm{H}$ ). The Dimer phase has perfect string order $\tilde{\mathcal{O}}_{X}=1$ corresponding to the $H D_{X}$ phase. At the transition, the order parameter $\tilde{\mathcal{O}}_{X}$ jumps from 1 to 0 , while $\tilde{\mathcal{O}}_{\perp}$ jumps from 0 to 0.84 , the value corresponding to the spin- 1 antiferromagnetic chain. 


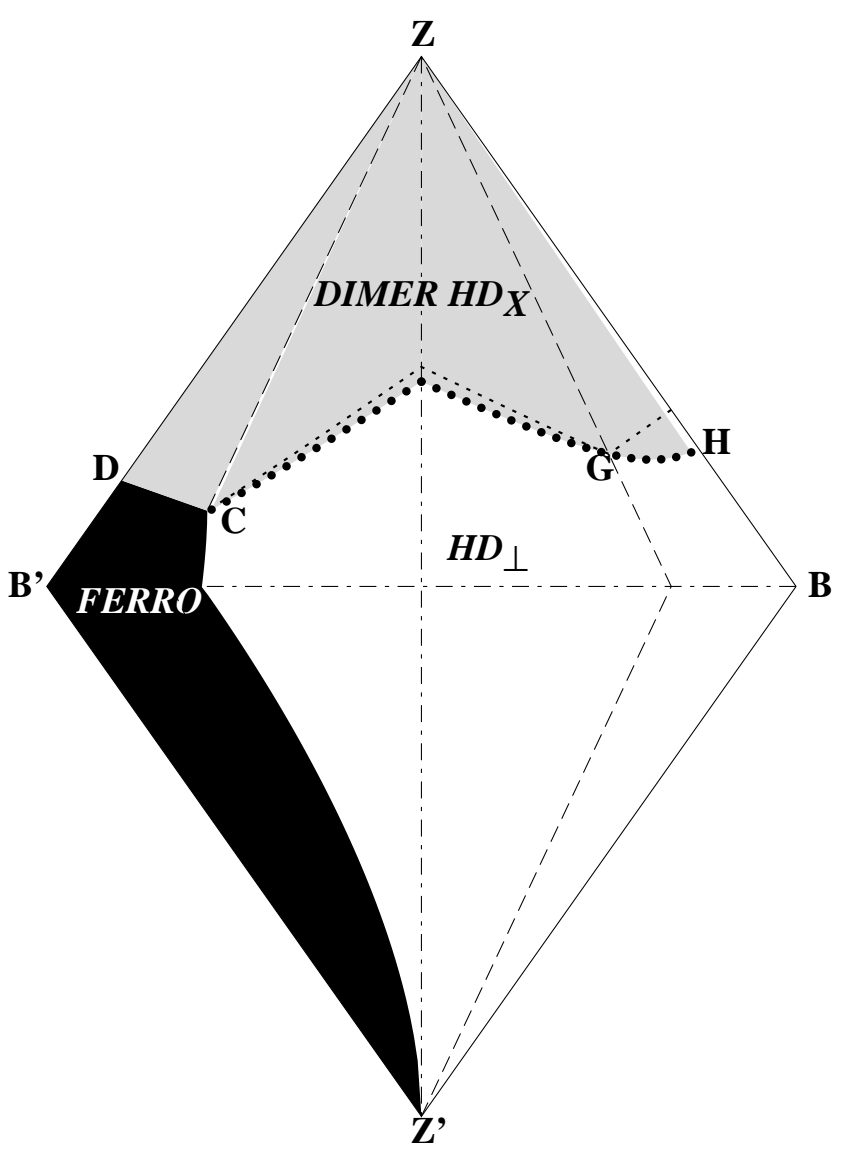

FIG. 7. The $\left(J_{X 1}+J_{X 2}\right)=2 J_{\|}$plane. The dashed lines correspond the intersection of this plane with the "Zig-Zag" model and G represents the Majumdar-Ghosh point. The black area represents the ferromagnetic region, whose boundaries are exactly known. The line Z'BZ represents the "composite-spin" model: $J_{X 1}=J_{X 2}=J_{\|}$. At $H$, there is a first order transition from the Haldane $H D_{\perp}$ phase to an exact Dimer phase which has the same topological long-range order as the $H D_{X}$ phase. The heavy dotted line CGH represents the first order transition between the Dimer Haldane phase $H D_{X}$ (grey area) and the Haldane $H D_{\perp}$ phase. The thin dotted line is an exact inner bound to the exact dimer phase. $C=(-2 / 7,-2 / 7,1 / 7)$ is an exact Triple point.

We have calculated the whole transition line $C G H$ between the $H D_{\perp}$ phase and $H D_{X}$ dimer phase through the DMRG scheme explained in section III. The transition is first order all along this line and the point $\mathrm{C}$ $=(-2 / 7,-2 / 7,1 / 7)$ appears as an exact triple point. Note that the Majumdar-Ghosh point $\mathrm{G}$ belongs to this line and is also an exact transition point. From $\mathrm{C}$ to $\mathrm{G}$ a trivial but remarkable exact inner bound to the Dimer state is obtained when the dimer energy $\lambda_{1}$ ceased to be the lowest eigenvalue for an isolated plaquette, i.e when $\lambda_{1}=\lambda_{6}$ (see Eq. 20). With our energy scaling [Eq. (3)], this lower bound corresponds to

$$
J_{\perp}=\left(-2+5 \mathcal{S}+\sqrt{8-36 \mathcal{S}+41 \mathcal{S}^{2}}\right) / 2
$$

for $\mathcal{S}>0$ and

$$
J_{\perp}=\left(-2-3 \mathcal{S}+\sqrt{8+28 \mathcal{S}+25 \mathcal{S}^{2}}\right) / 2
$$

for $\mathcal{S}<0$ (see the thin dotted line in Fig. 7). Beyond G, The upper bound to the dimer phase corresponds to the intersection of $\lambda_{1}$ and $\lambda_{3}$, i.e. to the segment $J_{\perp}=\mathcal{S}$.

\section{B. The general phase diagram}

We shall consider successively the 8 sectors of the tetrahedron XX'YY'ZZ' with the notation $\left[\epsilon_{x}, \epsilon_{y}, \epsilon_{z}\right]$, where $\epsilon_{\alpha}= \pm$ represents the sign of the coordinate $\alpha$

\section{The $[+,+,+]$ sector, with $J_{\|}, J_{\perp}$ and $\mathcal{S}$ positive}

\section{a. The boundary face $X Y Z$}

The boundary face XYZ, with equal antiferromagnetic diagonal interactions $J_{\mathrm{x} 1}=J_{X 2}=J_{X}$ [Fig. 1 (b)], has been studied by Wangl. There is an obvious symmetry which exchanges $J_{\|}$and $J_{X}$ by twisting every other rung by an angle $\pi$ around the axis of the ladder. ZB is a symmetry line. It is thus sufficient to study the half triangle XBZ. From the recent field-theoretical results of Kim et al.10, near X, which represents two decoupled spin- $\frac{1}{2}$ chains, there is a first order transition between the two Haldane phases $H D_{X}$ and $H D_{\perp}$ along the line $J_{\perp}=\mathcal{S}$. From the previous paragraph there is a first order transition between the same phases along the symmetry axe $\mathrm{ZB}$ at $H$. Using the DMRG algorithm described in section III, we have calculated the full transition line from $\mathrm{X}$ to $H$. It is show in Fig. 8. It is in good agreement with earlier results obtained by Wang 0 . However, in contrast to the conclusions of Wang, our results show that all along this line we have a first-order transition with finite gap from the $H D_{X}$ to the $H D_{\perp}$ Haldane phase, in agreement with theoretical-field result 10 .

As pointed out in section III, in contrast to our algorithm which uses different boundary conditions on either side of the transition line to avoid the degeneracy of the ground state, Wang has used everywhere the DMRG algorithm with boundary conditions corresponding to Fig. 5 (a). 


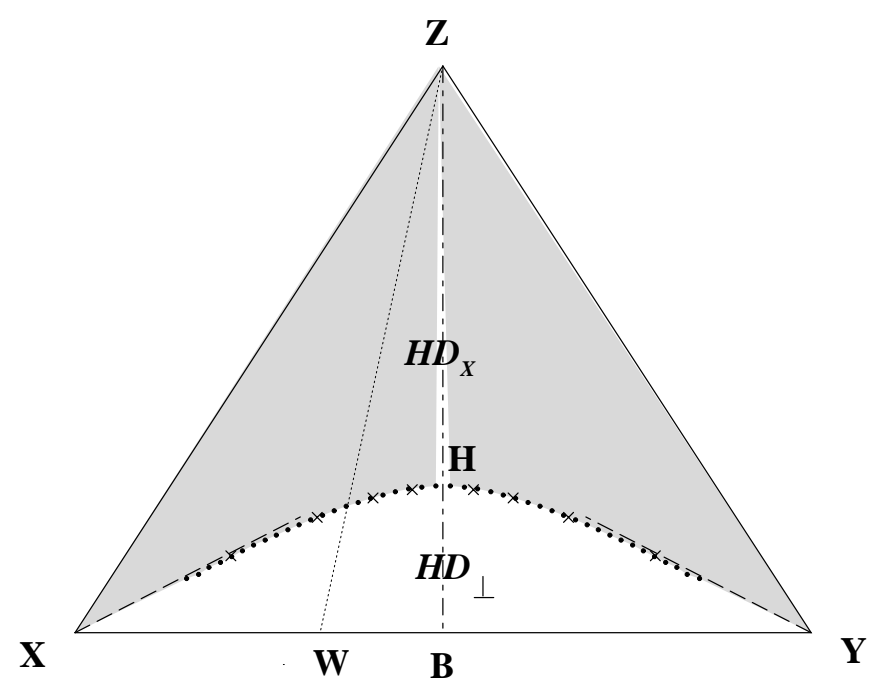

FIG. 8. The XYZ face with equal diagonal interactions and all $J$ 's positive. The symmetry axis ZB corresponds to the "composite spin" model with a first-order transition from the Haldane $H D_{\perp}$ phase to the dimer $H D_{X}$ phase at $H$. The dashed lines are exact first order transition lines between the $H D_{\perp}$ and $H D_{X}$ phase in the neighborhoods of $\mathrm{X}$ and $\mathrm{Y}$ (two independent spin- $\frac{1}{2}$ chains) obtained through field-theoretical methods). The dots are our first order transition points obtained through DMRG. They are compared to earlier results of Wang (crosses). The behavior of nearest-neighbor pair correlations, gap and string-order parameter along the lines ZB and ZW corresponding to $J_{\|}=J_{X 1}+J_{X 2}$ are shown in Fig. 9

As he observed, below the transition line, the ground state is degenerate and it is nondegenerate above this line. However, his conclusion suggesting a transition from a Haldane phase to a "Dimer" phase with different properties concerning the behavior with respect to boundary conditions is incorrect. If we use everywhere the DMRG algorithm with the other boundary condition corresponding to Fig. 5 (b) we find a degenerate ground state above the transition line and a non-degenerate ground state below! The correct interpretation is, as previously stressed, the transition between two topologically distinct Haldane phases. Typical sets of curves showing the discontinuities in the first-neighbor pair correlations [Eq. (11)], gap and string-order parameters along the dotted line ZW in Fig. 8 corresponding to $J_{\|}=J_{X 1}+J_{X 2}$ and along the symmetry line ZB are shown in Fig. 9.

\section{b. The $O Y Z$ plane}

The OYZ $\left(J_{\|}=0\right)$ plane corresponds to coupled dimerized chains. In this plane (Fig. 10) the point L corresponds to a simple antiferromagnetic Heisenberg chain and Y represents two decoupled Heisenberg chains. Strong arguments have been given for the existence of a critical line joining L to $\mathrm{Y}_{2} 3 \mathrm{2} 4$ and a simple analytic approximation of this line has also been conjectureden. (a)

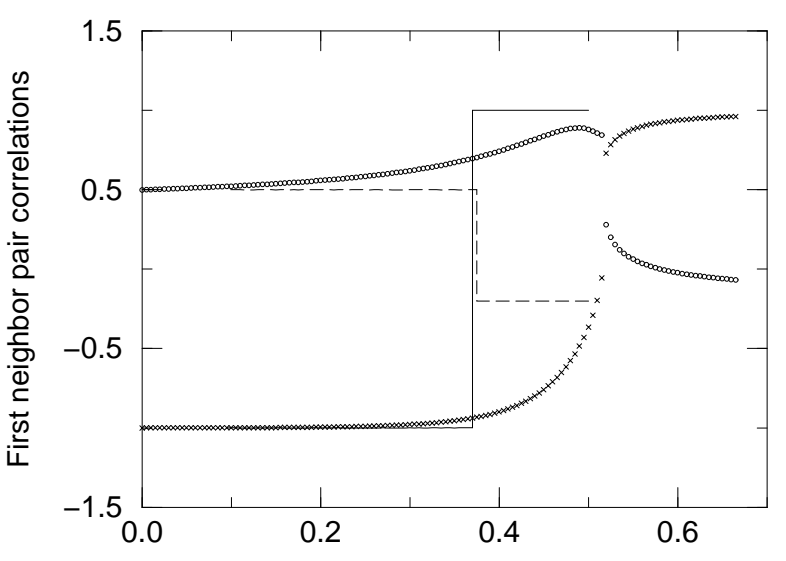

(b)

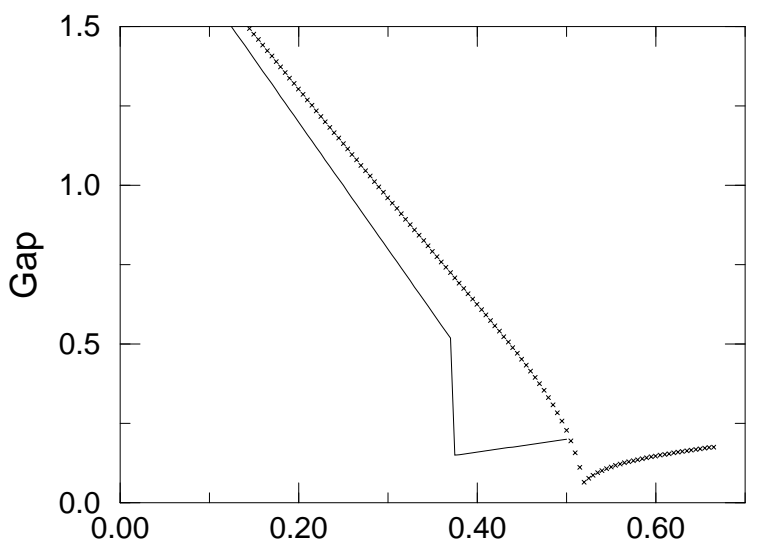

(c)

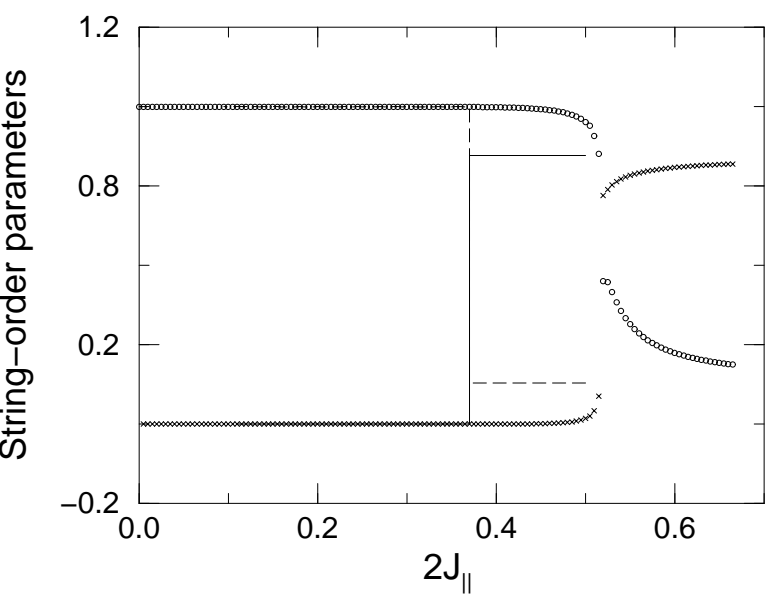

FIG. 9. First order transition between Haldane phases with different topological order. (a) Pair correlation $C_{\perp}$ along ZW (crosses) and ZB (full line); pair correlation $C_{X 1}$ along ZW (circles) and ZB (dashed line). (b) Gap along ZW (crosses) and ZB (full line). (c) String order parameter $\tilde{\mathcal{O}}_{X}$ along ZW (crosses) and ZB (full line); string order parameter $\tilde{\mathcal{O}}_{\perp}$ along ZW (circles) and ZB (dashed line). 


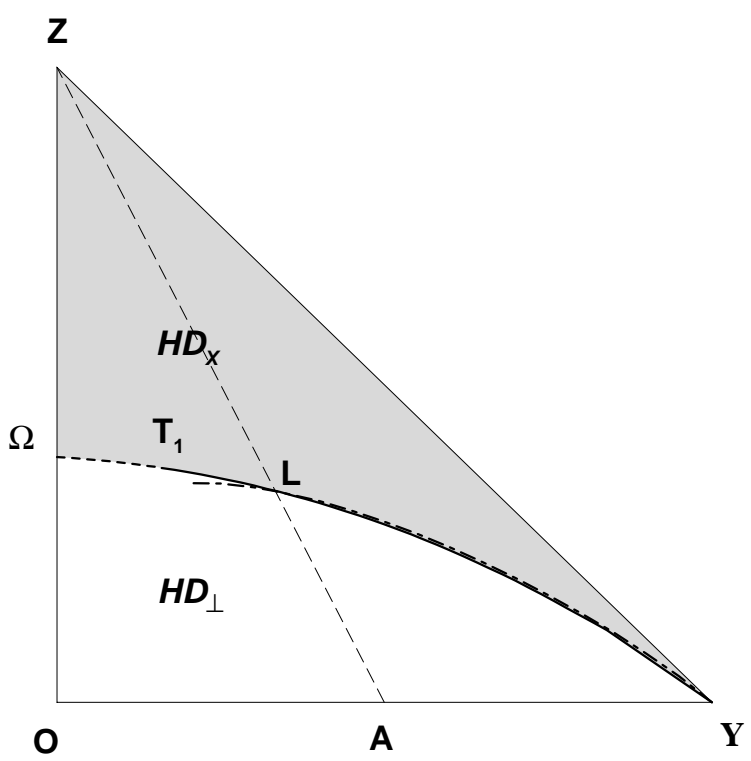

FIG. 10. The transition line between the two Haldane phases with different topological order in the $J_{\|}=0$ plane. L represents a simple spin- $\frac{1}{2}$ antiferromagnetic Heisenberg chain and $\mathrm{Y}$ two decoupled chains. The heavy line $\mathrm{YLT}_{1}$ is critical with zero gap. On $\mathrm{T}_{1} \mathrm{~L}$ (dotted line), the transition is first order. The dash-dotted line is a simple approximation to the critical line.

With our nontrivial energy scaling [Eq. (3)], it corresponds to the real root of the third-order equation:

$$
J_{\perp}^{3}-\mathcal{S}\left(1-J_{\perp}\right)^{2}-\mathcal{S}^{3}+2 \mathcal{S}^{2}\left(1-J_{\perp}\right)=0
$$

We have determined through DMRG the transition between the two Haldane phases in this plane (see Fig. 10). From $\mathrm{X}$ to $\mathrm{L}$, the transition line follows approximately the analytic form previously proposed but strongly differ beyond $\mathrm{L}$. The critical line extends beyond $\mathrm{L}$ up to a point $T_{1}$ that we have determined through the same method as that used for the "Zig-Zag" model (cf. section IV). From $T_{1}$ to $\Omega$, the transition is first order with finite gap.

\section{c. The transition surface}

We have exhibited four transition lines, between the two Haldane phases with different topological order, in four different planes of the phase diagram: XAZ, XYZ, OBZ, OYZ. We expect that all four lines belong to a same transition surface. Using the DMRG algorithm described in section III, we have investigated this transition surface over a fine grid in the $\{\mathrm{x}, \mathrm{y}\}$ plane. As a good compromise, we kept 30 to 60 states in the DMRG scheme and chose a $0.02 \times 0.02\{\mathrm{x}, \mathrm{y}\}$ grid.

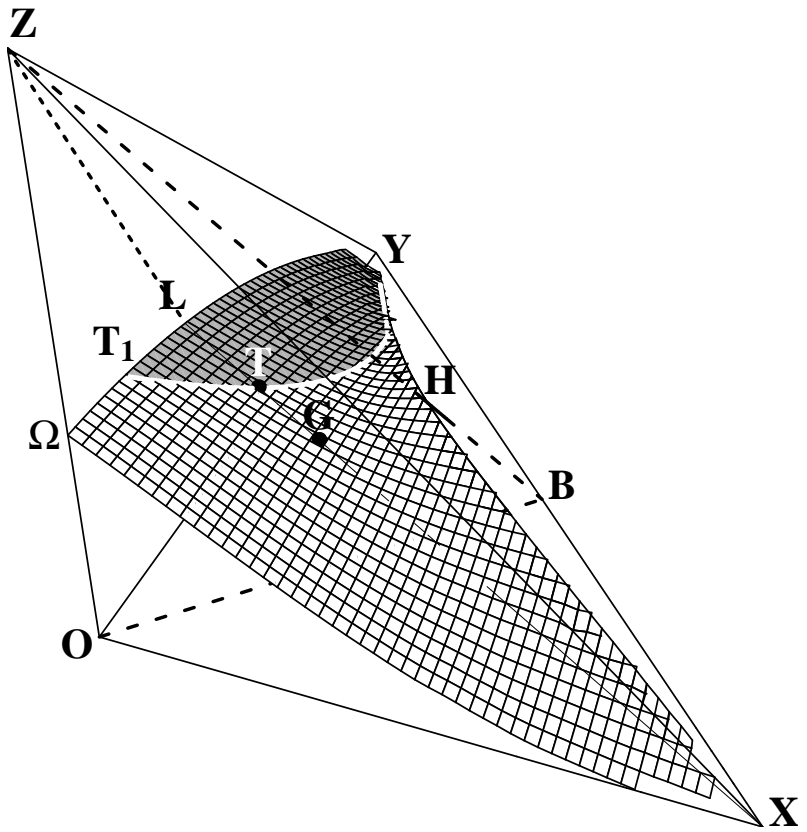

FIG. 11. The transition surface separating the two gapped Haldane phases with different topological order in the $[+,+,+]$ sector. Each point of the grid has been obtained through DMRG, keeping 30 states. The dark part of the surface is critical with vanishing gap and string-order parameters. The clear part corresponds to a first-order transition with finite gap and degenerate ground state. The white line which separates both parts of the surface thus appears as a tricritical line. The cuts of the surface through the planes XALZ, OBHZ, $\mathrm{XYZ}$, and OYZ have been represented in Figs. 6, 7, 8 and 10 respectively. The straight line LTGX, joining L (one simple Heisenberg chain) to X (two decoupled Heisenberg chains) lies in the surface. The Majumdar-Ghosh point $\mathrm{G}$ corresponds to the intersection of this line with the OBHZ plane. Near X, our DMRG results are consistent with the exact result obtained from field theory (see text), i.e. the transition surface is tangent to the plane $J_{\perp}=\mathcal{S}=J_{X 1}+J_{X 2}$.

The results are shown in Fig. 11. The cuts of this surface through the planes XALZ, OBHZ, XYZ and OYZ have already been represented in Figs. 6, 7, 8 and 10 respectively. The straight line joining $\mathrm{L}$ (simple antiferromagnetic spin- $\frac{1}{2}$ Heisenberg chain) to $\mathrm{X}$ (two decoupled Heisenberg chains) is a symmetry line in the AXZ plane (cf. section IV) and lies in the transition surface. We used it for an accuracy test on our DMRG algorithm. For the points of our $\{\mathrm{x}, \mathrm{y}\}$ grid which lie on the projection of this line in the XOY plane, we have plotted in Fig. 12 the difference $\left|z_{c}^{D M R G}-z_{c}^{\text {Exact }}\right|$ between the approximate coordinate $z_{c}^{D M R G}$ of the surface obtained through DMRG and the exact value $z_{c}^{\text {Exact }}$ corresponding to LX. 


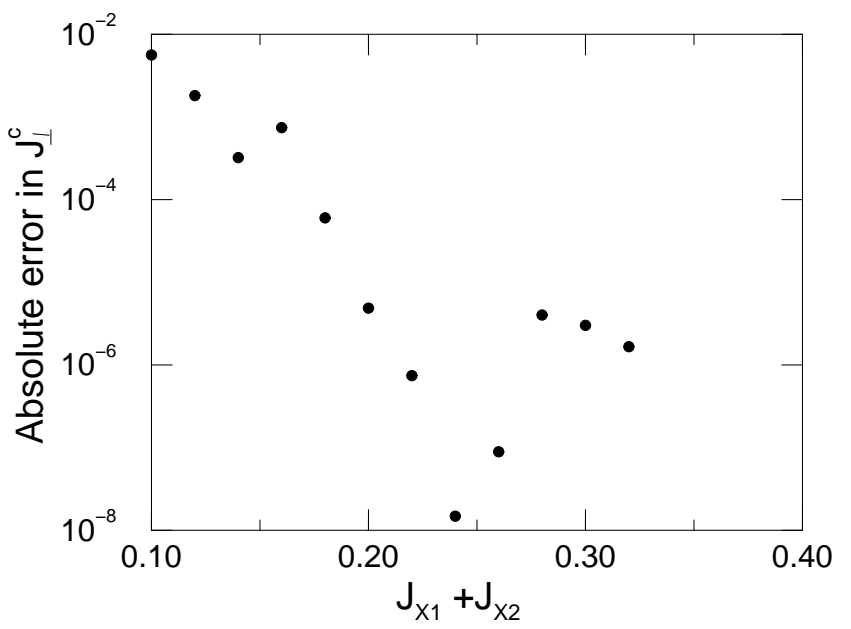

FIG. 12. A test of accuracy of our DMRG scheme, with only 30 states kept, along the exact transition line LX. The accuracy is best ( 7 digits) near the Majumdar-Ghosh point $G$. It decreases drastically to 3 to 2 digits close to $\mathrm{X}$ (two decoupled Heisenberg chains) and also decreases when moving to $\mathrm{L}$ in the critical regime as the second-order transition becomes softer.

Except in the neighborhood of X: two decoupled spin- $\frac{1}{2}$ Heisenberg chains, where a much larger number of states $\approx 30^{2}$ should be retained in the DMRG scheme to obtain precise results, we obtain an accuracy which is better than three to four digits on $z_{c}$. The accuracy is the best (7 digits) near the Majumdar-Gosh point G. It decreases drastically to $3 \sim 2$ digits close to $\mathrm{X}$ (two decoupled Heisenberg chains) and is of about 5 digits near $\mathrm{L}$, in the critical regime.

Field-theoretical results 10 prove that in both planes $\mathrm{XYZ}$ and XALZ the transition line, near $\mathrm{X}$, is first order and correspond to $\mathcal{S}=J_{\perp}$. In agreement with these exact results, our numerical data indicate that the transition surface is tangent at X to the plane $\mathcal{S}=J_{\perp}$.

Knowing this transition surface, we now determine which part of it is critical, with zero gap. We already know two intersecting critical lines $\mathrm{LT}_{1}$ and YLT. We expect that they lie in a same critical part of the surface which is limited by a line of tricritical points ("tricritical line") passing through $\mathrm{T}$ and $\mathrm{T}_{1}$. To determine this tricritical line, we choose the same methods as these previously used for the "Zig-Zag chain" model17.18 (see section IV).

It is proved from field theory that, when umklapp interactions become relevant variables, simultaneously the spectrum develops a gap between the $\mathrm{S}=0$ ground state and the first excited $\mathrm{S}=1$ states and also the degeneracy of the first $\mathrm{S}=0$ excited state and lowest $\mathrm{S}=1$ state is split. Hence in the thermodynamical limit, the difference $\delta$ between the energies of these two lowest excited states is zero at the critical value for exchange parameters where the gap vanishes. As a simple illustration, let us consider the curved line $\Omega L Y$ (Figs. 10 and 11) corresponding to the cut of the transition surface through the OYZ plane.

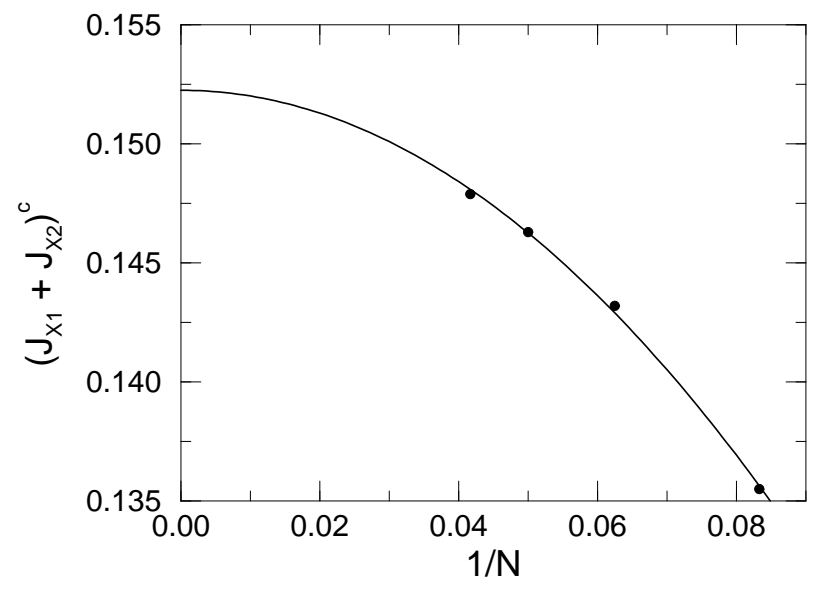

FIG. 13. Critical value of $\mathcal{S}=J_{X 1}+J_{X 2}$ for which the difference $\delta$ between the two lowest excited states vanishes, as a function of $1 / \mathrm{N}$, for finite chains with periodic boundary conditions. We follow the transition line $\Omega L Y$ determined through DMRG in the OYZ plane (Fig. 10). A quadratic extrapolation gives $\left(J_{X 1}+J_{X 2}\right)^{c} \approx 0.152$ in the thermodynamic limit, fixing the position of $\mathrm{T}_{1}$ on the transition line.

Moving along this curve, we use Lanczös diagonalisation on finite systems of length $L=N / 2$, with periodic boundary conditions, to calculate the value $\mathcal{S}_{c}(N)$ of the parameter $\mathcal{S}=J_{X 1}+J_{X 2}$ at which $\delta$ vanishes.

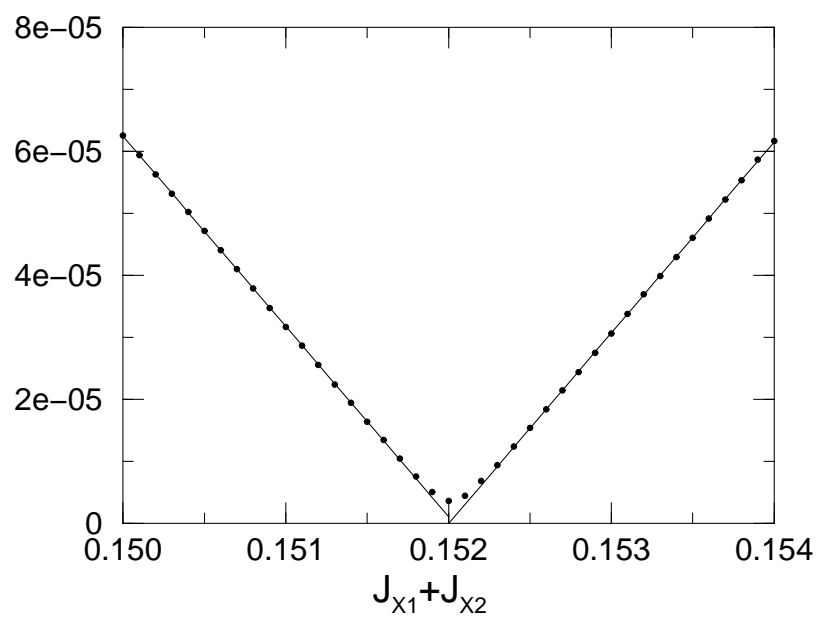

FIG. 14. Mean square deviation with respect to a $1 / N^{3}$ law of the difference between the two first excited levels of a finite ladder with periodic boundary conditions. We follow the cut of the transition surface through the plane $2 J_{\|}=0$ determined through DMRG. 
The results are represented in Fig. 13 as a function of $1 / N$. A quadratic extrapolation gives $\mathcal{S}_{c}(N \rightarrow \infty) \approx$ 0.152 in the thermodynamic limit.

A much better accuracy (six digits) on the position of $\mathrm{T}$ on LX, for the "Zig-Zag chain" model has been obtained recently by Eggert18. He used the field-theoretical result that, on LX at the critical point $\mathrm{T}$, the marginal operator vanishes and the energy difference $\delta(N)$ between the two first excited levels is exactly proportional to $1 / N^{3}$. Although we have not been able to generalize this fieldtheoretical result for every point on the tricritical line, we have found numerically that except in the vicinity of the boundary plane XYZ, this $1 / N^{3}$ law works remarkably well for a large part of the tricritical line. And we have used it to improve our accuracy.

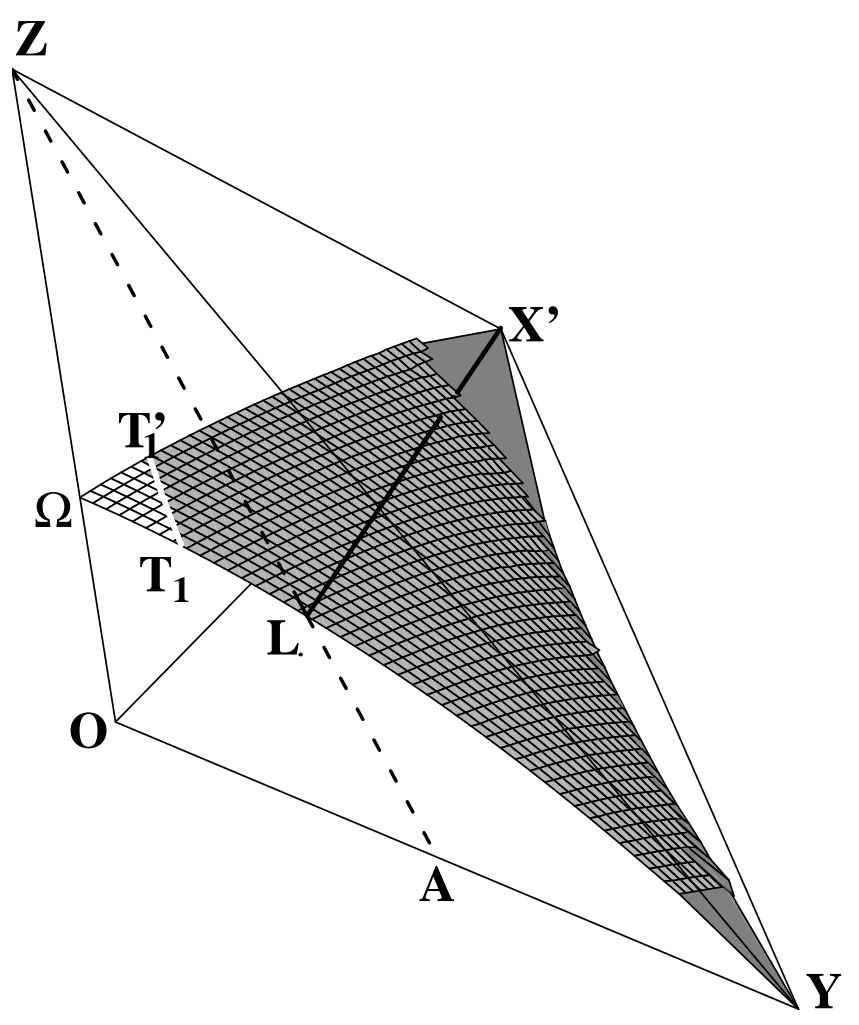

FIG. 15. The transition surface between the two Haldane phases with different topological order in the $[-,+,+]$ sector. The dark part of the surface corresponds to the zero-gap critical part. All points of the mesh are DMRG results. Our DMRG result are not accurate in the vicinity of ferromagnetic (X') or antiferromagnetic (Y) decoupled chains. The grey areas are simple extrapolations. The critical part of the surface is bounded through the tricritical $\mathrm{T}_{1} \mathrm{~T}_{1}{ }_{1}$ white line. On $\mathrm{T}_{1} \mathrm{~T}_{1} \Omega$ the transition is first order with two degenerate ground-states and finite gap. The cut by the plane ALZX' corresponds to the "Zig-Zag chain" model (cf. Fig. 6 in section IV). The straight line LX' lies in the critical surface.
Figure 14 illustrates around the critical value $\mathcal{S}=$ 0.152 found by the previous method (Fig. 13) the mean square deviation of $\delta(N)$ with respect to a $1 / N^{3}$ law, when we follow the transition line determined through DMRG in the OYZ plane. The results are based on Lanczös diagonalizations on finite systems with $\mathrm{N}=16$, 20 and 24 sites. The critical value thus obtained $\mathcal{S}=$ 0.15201 is improved up to five digits. This corresponds to the accuracy on the position of the transition line obtained through DMRG (see Fig. 12).

Taking successive cuts of the transition surface through different vertical planes at $J_{\|}=C^{s t}$. or $\mathcal{S}=C^{s t}$. and using the same methods, we have determined the whole tricritical line $\mathrm{T}_{1} \mathrm{TY}$ representing the boundary of the critical part of the transition surface (heavy white line in Fig. 11). Near Y, this line is close to the boundary of the surface of transition in the XYZ plane. We do know however that it does not intersect it: exact field-theoretical result 10 prove that in the XYZ plane the transition is first order near Y.

$$
\text { 2. The }[-,+,+] \text { sector }
$$

The transition surface and its zero-gap critical part is determined though the same methods as used in the previous subsection. The results are shown in Fig. 15. The dark part of the surface is critical with zero gap. It does not intersect the $\mathrm{X}^{\prime} \mathrm{YZ}$ boundary face and extends further in the $[-,+,-]$ sector. It is bounded through the tricritical line $\mathrm{T}_{1} \mathrm{~T}_{1}^{\prime}$. On the left of this line $\left(\mathrm{T}_{1} \mathrm{~T}_{1}{ }_{1} \Omega\right)$ part, the transition is first order with degenerate ground state and finite gap. The cut through the ALZX' plane corresponds to the "Zig-Zag chain" model (see Fig. 6 in section IV). The LX' straight line is a symmetry line in that plane and it lies in the critical surface.

$$
\text { 3. The }[-,-,+] \text { sector }
$$

This sector is particularly interesting since the two Haldane phases with different topological order compete here with the ferromagnetic phase.

\section{a. The ferromagnetic volume}

From section IV A, the A'X'Z plane corresponding to the "Zig-Zag chain" model $\left(J_{X 1}=0\right)$ splits the ferromagnetic boundary in two parts. On the $J_{X 1}>0$ side the exact boundary of the ferromagnetic domain is the surface determined by Eq. (20), taking into account our energy scaling [Eq. (3)]. On the other side $\left(J_{X 1}<0\right)$, we have calculated the ferromagnetic boundary with an accuracy of three to four digits through DMRG (see Fig. 16). The straight line segment CD corresponding to the intersection with the OB'Z plane (cf. Fig. 7) lies in this boundary surface. Near $X^{\prime}$, we have two weakly coupled ferromagnetic chains. At first order in perturbation theory, our Hamiltonian can be written as: 


$$
H_{e f f}=L J_{\|}+\left(J_{\perp}+\mathcal{S}\right) \mathbf{S}_{\mathbf{T}}^{\mathbf{a}} \cdot \mathbf{S}_{\mathbf{T}}^{\mathbf{b}} / 2 L
$$

where

$$
\mathbf{S}_{\mathbf{T}}^{\mathbf{a}}=\sum_{i=1}^{L} \mathbf{S}_{\mathbf{2}} \quad \text { and } \quad \mathbf{S}_{\mathbf{T}}^{\mathbf{b}}=\sum_{i=0}^{L-1} \mathbf{S}_{\mathbf{2} \mathbf{i}+\mathbf{1}}
$$

represent the total spins of the two coupled ferromagnetic chain. We deduce that the plane $\left(J_{\perp}+\mathcal{S}=0\right)$ is tangent at $\mathrm{X}^{\prime}$ to the ferromagnetic surface.

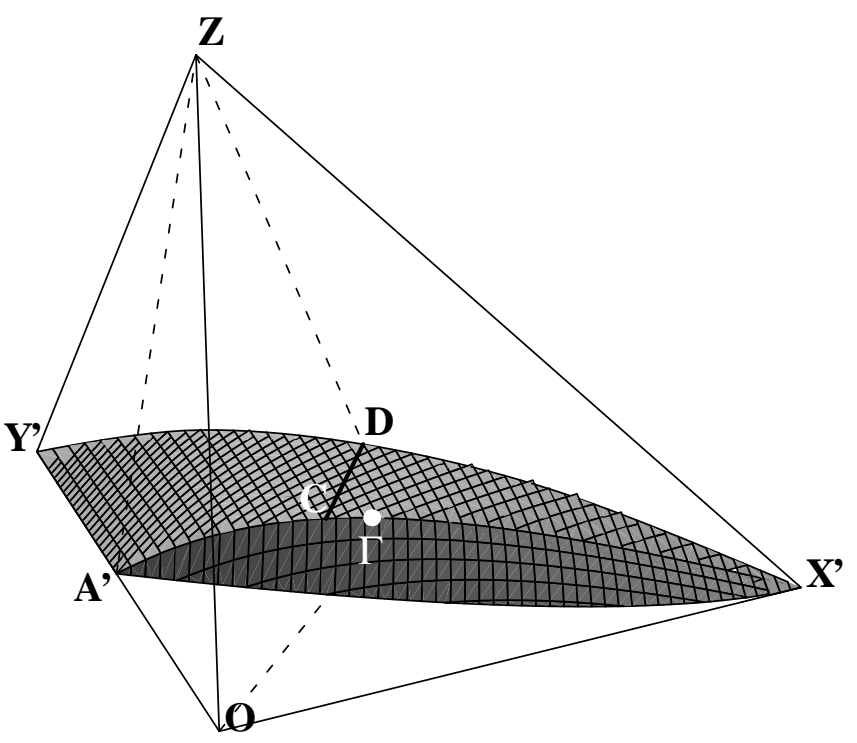

FIG. 16. The surface limiting the ferromagnetic phase in the $[-,-,+]$ sector. The line $\mathrm{A}^{\prime} \mathrm{C} \Gamma \mathrm{X}^{\prime}$ at the intersection of this surface with the $\mathrm{A}^{\prime} \mathrm{X}^{\prime} \mathrm{Z}\left(J_{X 1}=0\right)$ plane corresponds to the exactly soluble DKO model. The front part of the surface corresponding to $\left(J_{X 1}>0\right)$ is also known exactly [Eqs. $(3,20)]$. The back part of the surface corresponding to $\left(J_{X 1}<0\right)$ has been determined through DMRG. The straight line CD corresponding to its intersection with the OBZ plane (see Fig. 7) is exact. The line ACX' corresponding to the intersection of the surface with the A' $X^{\prime} Z$ plane is singular. From A' to $\Gamma$, our DMRG results show a discontinuity in the gradient to the surface. From $\Gamma$ to $\mathrm{X}$, there are singularities in higher derivatives. The Fig. 18 show that this line corresponds to the intersection of the ferromagnetic surface with the transition surface between the two Haldane phases with different topological order. $\Gamma$ is at the crossing of the A' $\mathrm{CX}$ ' line with the tricritical line limiting the critical part of this latter transition surface.

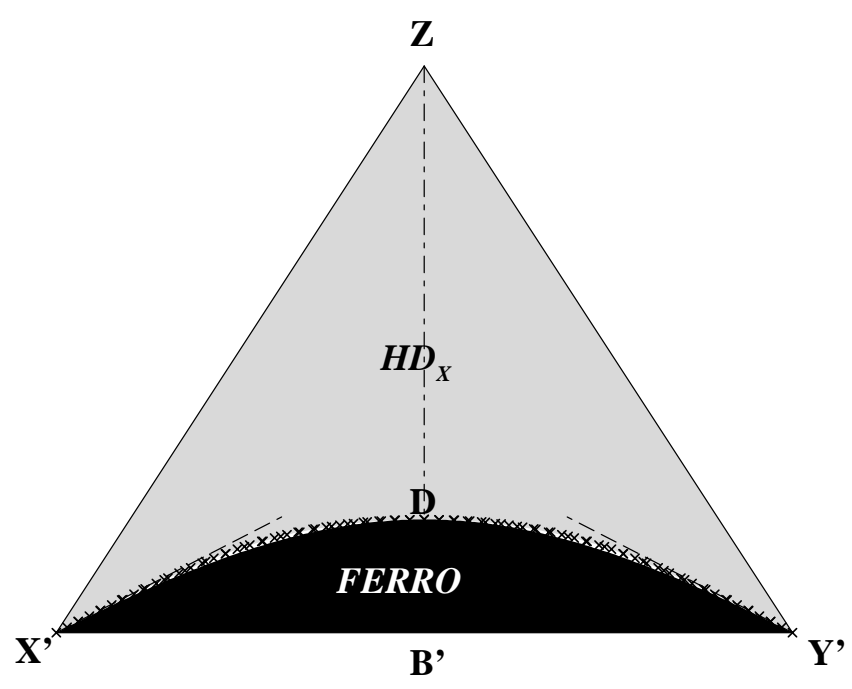

FIG. 17. The first order transition between the $H D_{X}$ and ferromagnetic phase in the $X^{\prime} Y^{\prime} Z$ face. The dashed lines are the cuts through the exact tangent planes to the ferromagnetic boundary at X' and Y' (two independent ferromagnetic chains). The dots are our DMRG results. the boundary of the black area represents a simple inner bound to the ferromagnetic region obtained through [Eqs. $(3,21)]$.

Similarly, the whole segment A'Y' of the OY' axis corresponds to two decoupled identical ferromagnetic chains with alternating $\left(J_{X 1}, J_{X 2}\right)$ ferromagnetic bonds. The segment A' $Y^{\prime}$ lies in the ferromagnetic boundary surface and near A'Y', at first order in perturbation theory the Hamiltonian can be expressed as:

$$
H_{e f f}=L \mathcal{S}+\left(2 J_{\|}+J_{\perp}\right) \mathbf{S}_{\mathbf{T}}^{\mathbf{a}} \cdot \mathbf{S}_{\mathbf{T}}^{\mathbf{b}} / 2 L
$$

where

$\mathbf{S}_{\mathbf{T}}^{\mathbf{a}}=\sum_{i=1}^{L / 2}\left(\mathbf{S}_{\mathbf{4 i}}+\mathbf{S}_{\mathbf{4 i}-\mathbf{3}}\right) \quad$ and $\quad \mathbf{S}_{\mathbf{T}}^{\mathbf{b}}=\sum_{i=0}^{L-1}\left(\mathbf{S}_{\mathbf{4 i}-\mathbf{1}}+\mathbf{S}_{\mathbf{4 i - 2}}\right)$

and the plane $\left(2 J_{\|}+J_{\perp}\right)=0$ is tangent to the ferromagnetic boundary all along the segment A'Y'.

Although the inner envelope to this boundary surface determined by Eqs. $(3,21)$ is not exact, it is everywhere an excellent approximation (see the comparison in the $\mathrm{X}^{\prime} \mathrm{Y}^{\prime} \mathrm{Z}$ plane represented in Fig. 17). This is due to the fact that three different exact transition lines: $\mathrm{A}^{\prime} \mathrm{Y}^{\prime}, C D$ and A'CXX' belong to this envelope and that the planes previously considered are also tangent to this envelope.

From our DMRG results, it is clear that the line A'СГX', at the intersection of the A'X'Z plane ("Zig-Zag chain" model) with the ferromagnetic surface and corresponding to DKO exact model is singular. On this line, there are discontinuities in the gradient to the surface from $\mathrm{A}$ to $\Gamma$, a point whose position will be given below. From $\Gamma$ to X', there are singularities in higher-order derivatives. 


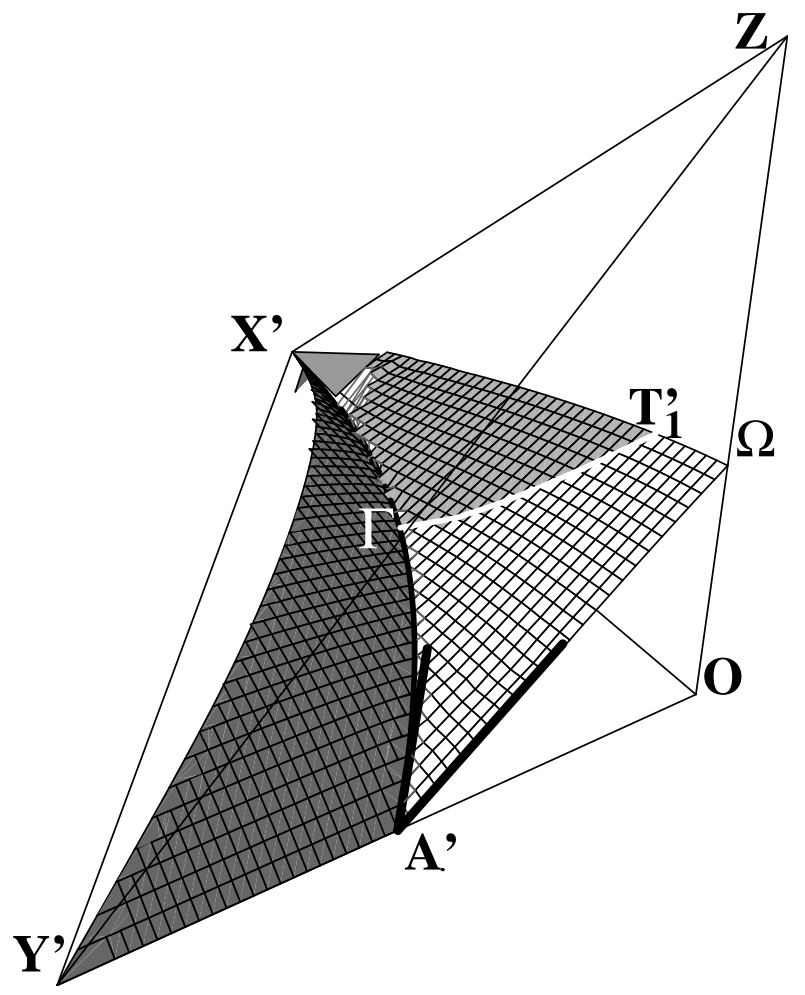

FIG. 18. Transition surfaces in the $[-,-,+]$ sector. The dark-grey surface A'X'Y' is the boundary of the ferromagnetic phase and corresponds to a first-order transition between the Haldane $H D_{X}$ and the ferromagnetic phase. The other surface corresponds to the transition between the two Haldane phases $H D_{X}$ and $H D_{\perp}$ with different topological order. The dark part of this surface is critical with zero gap and string order parameters. On the clear part, the transition is first order and the gap is finite. This surface abuts the ferromagnetic boundary along a line $A^{\prime} \Gamma X^{\prime}$ which lies in the AX'Z plane $\left(J_{X 1}=0\right)$ and corresponds corresponds to the exactly soluble DKO model. The white line $\mathrm{T}_{1}{ }_{1} \Gamma$ limiting the critical zero gap surface ends on the DKO line at $\Gamma$. The part $\mathrm{A}^{\prime} \Gamma$ of the DKO line is "Triple Line" while $\Gamma \mathrm{X}$ ' is a "Critical-End Line". The two heavy lines starting from A' represent the plane $J_{X 1}=0.7444\left(2 J_{\|}+J_{\perp}\right)$, where a first order transition between two Haldane phases with different topological order has been found by Kolezhuk et al. in the frustrated spin-1 chain. This plane is tangent to our transition surface.

\section{b. Triple line, critical-end line}

We have calculated the transition surface between the two Haldane phases with different topological order in the same way as in the other sectors (see Fig. 18)

To our numerical accuracy, we observe that the meeting of this surface with the ferromagnetic boundary occurs in the $\mathrm{X}^{\prime} \mathrm{A}^{\prime} \mathrm{Z}$ plane and corresponds to the DKO exactly-soluble model. The $\mathrm{X}^{\prime} \mathrm{T}{ }_{1} \Gamma$ part of this surface is critical with vanishing gap and string-order parame- ters. On the other part $\mathrm{A}^{\prime} \Gamma \mathrm{T}^{\prime}{ }_{1}$ the transition is first order and the antiferromagnetic ground-state is doubly degenerated.

The tricritical line $\mathrm{T}_{1} \Gamma$ limiting the critical zero-gap surface ends on the DKO line at a particular point $\Gamma$ :($0.35,-0.2518,0.1464)$. According to the usual terminology concerning three dimensional phase diagrams, the part $\mathrm{A}^{\prime} \Gamma$ of the DKO line is a line of triple points (i.e. a "Triple Line") while $\Gamma \mathrm{X}$ ' is a line of critical-end points (i.e. a "Critical-End Line").

Our numerical results concerning the coincidence of the meeting of the two surfaces with the DKO line are enforced by the following theoretical argument:

- In the region with $J_{X_{1}}<0$ all interactions except $J_{\perp}$ are ferromagnetic. Consequently singlet pairing ("valence bond") can only occur along the rungs, while triplet pairing can take place along diagonals. Consequently only the $H D_{X}$ Haldane phase can appear. We conclude that the transition surface between the two Haldane phases cannot cross the $\left(J_{X 1}=0\right) \mathrm{A}^{\prime} \mathrm{X}^{\prime} \mathrm{Z}$ plane. It only exists in the $J_{X_{1}}>0$ side.

- In the $J_{X_{1}}>0$ side, the ferromagnetic boundary is exactly known and corresponds to the simple Equation 20. This surface has no singularity in its gradient or higher order derivatives. The meeting of the two surface should correspond to a singularity on the ferromagnetic surface: discontinuity in the gradient for the first-order part of the surface, or singularities in higher order derivative for the critical part. Consequently the transition surface between the two Haldane phases do not touch the $J_{X_{1}}>0$ part of the ferromagnetic boundary.

- From the two previous remarks, we conclude that if the transition surface between the two Haldane phases meets the ferromagnetic surface, it can only be in the $\mathrm{A}^{\prime} \mathrm{X}^{\prime} \mathrm{Z}$ plane $\left(J_{X_{1}}>0\right)$ i.e. at the $\mathrm{DKO}$ line.

c. The neighborhood of the frustrated spin-1 Heisenberg chain

In section II A we have shown that the neighborhood of A' can be described through the frustrated spin-1 chain model (Eq. 5) with effective first-neighbor $J_{1}=2 J_{\|}+J_{\perp}$ and second-neighbor $J_{2}=J_{X 1}$ interactions. This model has recently been studied by Kolezhuk et al11 through DMRG. At a critical ratio $r_{c}=J_{2} / J_{1} \approx 0.7444$, there is a sharp discontinuity in the order parameter $\mathcal{O}_{X}$ suggesting a first order transition. This critical ratio corresponds here to the plane $J_{X 1}=r_{c}\left(2 J_{\|}+J_{\perp}\right)$. We have represented this plane in Fig. 18. It is tangent in $\mathrm{A}^{\prime}$ to our transition surface. Consequently our diagram also contains the results on the frustrated spin-1 Heisenberg chain, and the transition observed by Kolezhuk et al. corresponds to a first-order transition between the $H D_{X}$ and $H D_{\perp}$ phases. 


\section{The $[+,-,+]$ sector}

The transition surface between the two Haldane phases in the last of the four sectors with $J_{\perp}>0$ is represented in Fig. 19. Except in the thin tip close to $\mathrm{X}$ we have found no evidence for a zero-gap critical part. The poor accuracy of our DMRG results near X does not allow to be more quantitative. Again, near A', our calculated surface is tangent to the plane $J_{X 1}=0.7444\left(2 J_{\|} J_{\perp}\right)$ corresponding to the results of Kholezhuk et al.11 (see above)

An overall view of the four previous sectors corresponding to $J_{\perp}>0$ is shown in Fig. 20.

5. The $[-,-,-]$ sector with with $J_{\|}, J_{\perp}$ and $\mathcal{S}$ negative

In this sector the phase transitions are between the ferromagnetic and the $H D_{\perp}$ Haldane states. The corresponding surface is known exactly and corresponds to Eqs. $(3,20)$. It is shown in Fig. 21.

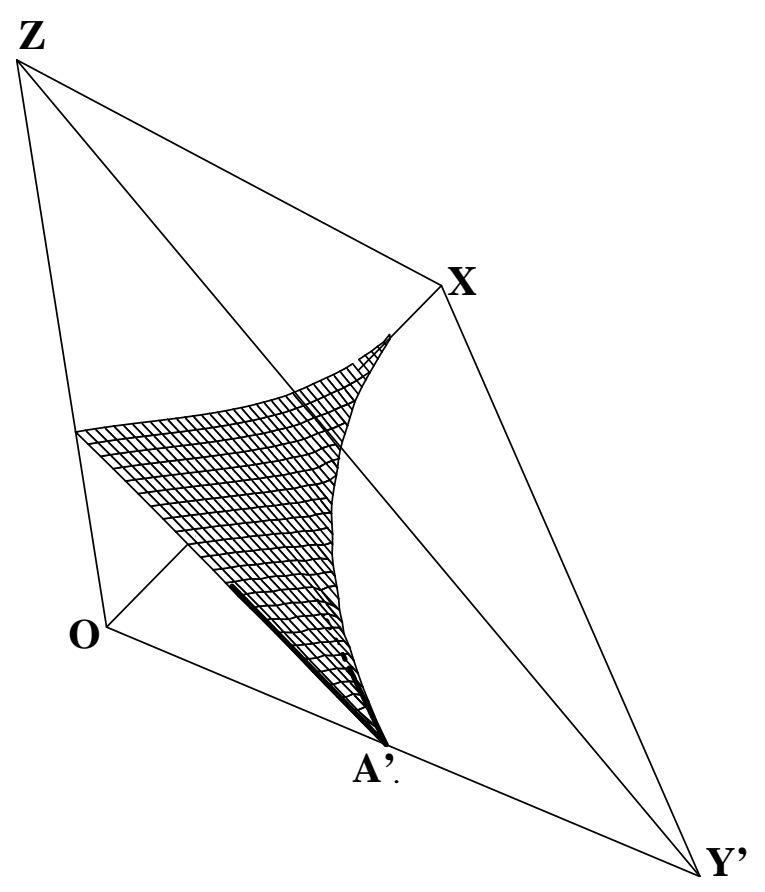

FIG. 19. The first order transition surface between the two Haldane phases $H D_{X}$ and $H D_{\perp}$ in the $[+,-,+]$ sector. The two heavy lines starting from A' represent the plane $J_{X 1}=0.7444\left(2 J_{\|}+J_{\perp}\right)$, where a first order transition between two Haldane phases with different topological order has been found by Kolezhuk et al. in the frustrated spin- 1 chain. This plane is tangent to our transition surface

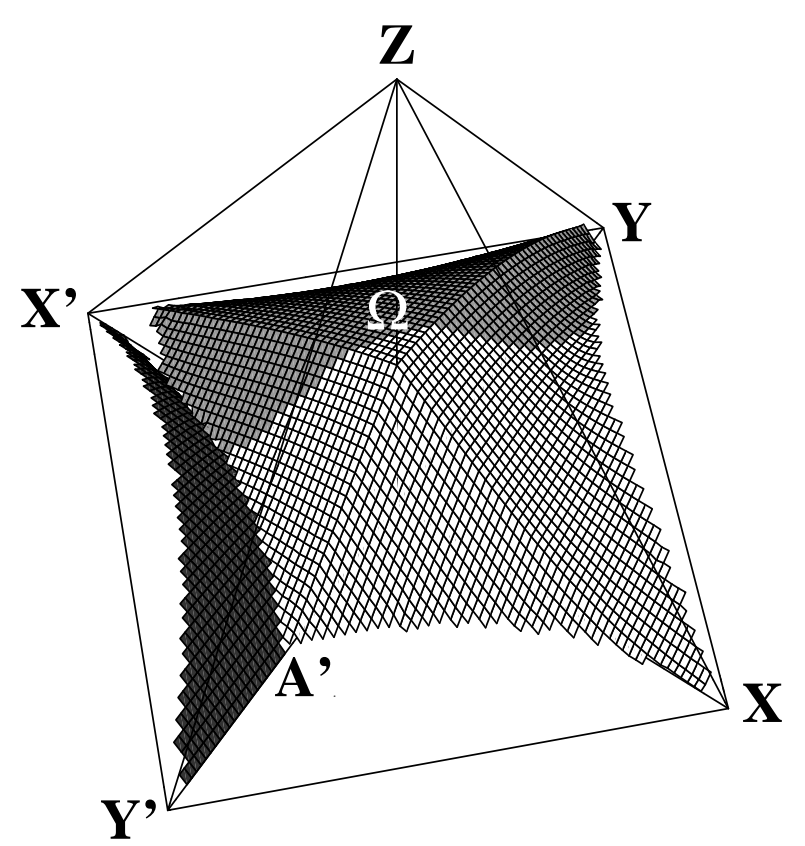

FIG. 20. An overall view of the transition surface in the upper half space. The black surface A'X'Y' represents the boundary of the ferromagnetic phase. The other surface represents the transition between the two Haldane phases $H D_{X}$ and $H D_{\perp}$. The dark part is critical with zero gap, the clear part corresponds to a first-order transition with finite gap. Singularities on the lines $\Omega \mathrm{A}, \Omega \mathrm{X}, \Omega \mathrm{Y}$, and $\Omega \mathrm{X}^{\prime}$ are not physical: they are related to our normalization condition (Eq. 3).

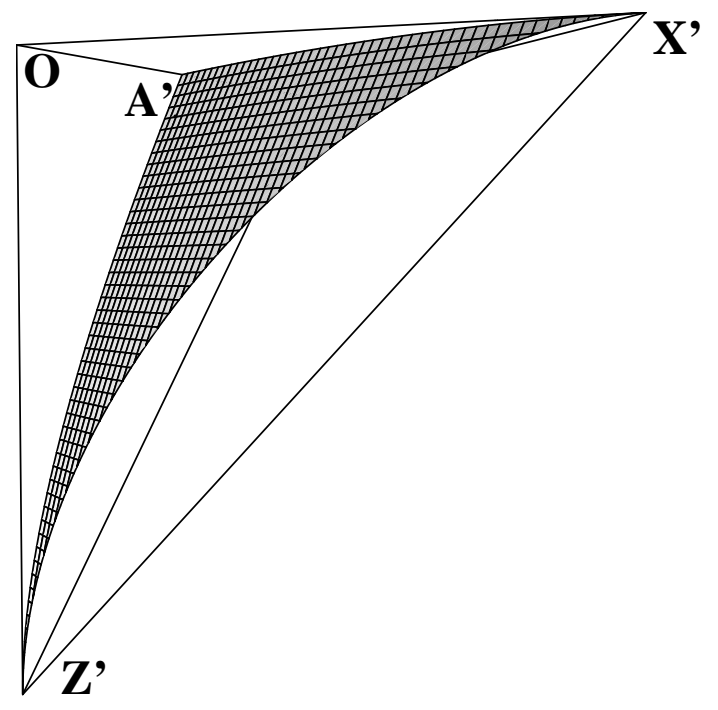

FIG. 21. The exact limit of the ferromagnetic phase in the $[-,-,-]$ sector 


\section{The $[+,-,-]$ sector}

The boundary face XY'Z' of this sector corresponds to the model represented in Fig. 1 (b) with ferromagnetic equal diagonal interactions and antiferromagnetic $J_{\|}$interactions. There is an obvious symmetry by exchanging the parallel and diagonal interaction which is obtained by twisting every other rung by an angle $\pi$ around the longitudinal axis of the ladder. Hence there is a one to one correspondence from each point of this face to a point of the YX'Z' face in the $[-,+,-]$ sector. The two-dimensional phase-diagram of the XY'Z' face is represented in Fig. 22. The transition line XE' between the two Haldane phases $H D_{X}$ and $H D_{\perp}$ is critical. Near X this critical line corresponds to $J_{\perp}=\mathcal{S}$ in agreement with field-theoretical results 10 . The critical line remains close to this asymptotic result except in the neighborhood of the ferromagnetic region, near E'.

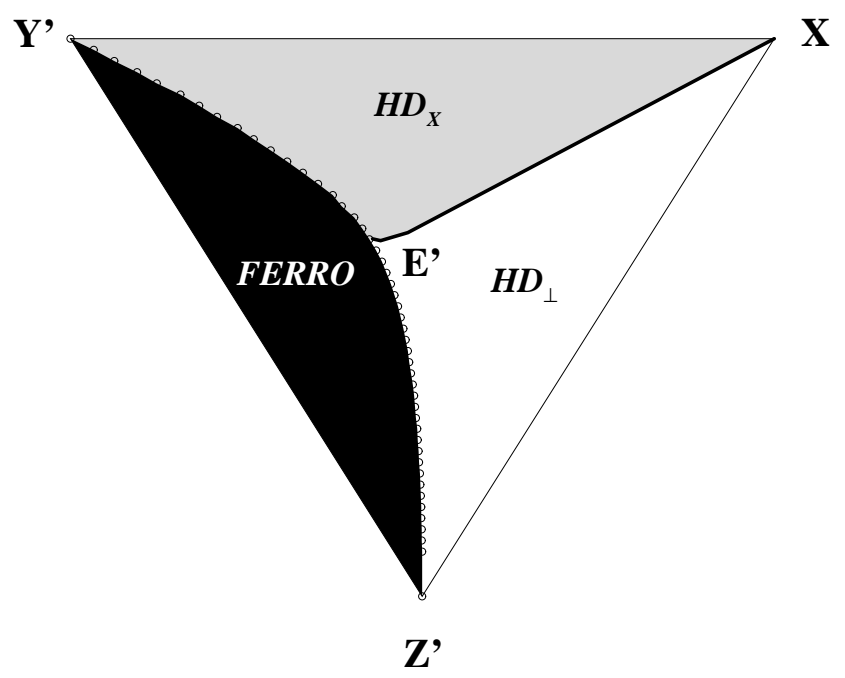

FIG. 22. The two-dimensional phase diagram in the boundary face $\mathrm{XY}^{\prime} \mathrm{Z}^{\prime}$ corresponding to equal diagonal interactions [Fig. 1 (b)]. The diagonal interactions are ferromagnetic while $J_{\|}$is antiferromagnetic. There is a symmetry which exchanges parallel and diagonal interaction by twisting every other rung by an angle $\pi$ around the longitudinal axis. Consequently the phase diagram of the YX'Z' face is identical. The transition line XE' between the two Haldane phases $H D_{X}$ and $H D_{\perp}$ is critical and remains close to its asymptotical limit at $\mathrm{X}$ $\left(J_{\perp}=\mathcal{S}\right)$ obtained from field theory, except near its meeting with the ferromagnetic boundary at E'.
The three-dimensional phase-diagram of the $[+,-,-]$ sector is shown in Fig. 23. The dark surface represents the ferromagnetic boundary seen from inside. It cuts the $\left(J_{X 1}=0\right)$ A'XZ' plane at the A'C'Z' line corresponding to the exactly soluble DKO model. The part of this surface which corresponds to $\left(J_{X 1}>0\right)$ is known exactly from Eq. (20). The other part is calculated through DMRG. The other surface represents the transition between the two Haldane phases $H D_{X}$ and $H D_{\perp}$. The clear part corresponds to a first order transition with finite gap and string-order parameter. The other part is critical with vanishing gap and string-order parameter. The blank tricritical line bounding this critical part has been determined through the methods explained above. Its intersection with the XOY' plane corresponds to $2 J_{\|} \approx 0.75$ and the critical surface has a small extension in the previous $[+,-,+]$ sector around $\mathrm{X}$. However our DMRG scheme is inadequate to determine the critical surface so close to X (two decoupled chains) with reasonable accuracy (see Fig. 19).

The symmetry line XC', in the $\left(J_{X 1}=0\right)$ A'XZ' plane lies in the transition surface (see Fig. 6b). C' represents the intersection of the line $\mathrm{XL}$ ' joining $\mathrm{X}$ (two decoupled antiferromagnetic chains) to L' (one ferromagnetic chain) with the DKO line. The surface starts at the $J_{\perp}=0$ plane roughly perpendicular to it and it curves sharply around $\mathrm{XC}$ ' to meet the boundary plane $\mathrm{XY}$ 'Z' along XE', nearly tangentially to the $\mathcal{S}=J_{\perp}$ plane. It abuts the ferromagnetic boundary along the $\mathrm{A}^{\prime} \mathrm{C}$ ' $\mathrm{E}$ ' curve, which is represented from inside the ferromagnetic region by a heavy line in Fig. 23.

The part A'C' of this curve lies in the $\mathrm{A}^{\prime} \mathrm{XZ}$ ' plane and coincides with the DKO curve. Since it corresponds to the meeting of two first-order transition surfaces, the curve A'C' is a triple line. This is justified by the following arguments:

- The transition surface between the two Haldane phases crosses the $\left(J_{X 1}=0\right) \mathrm{A}^{\prime} \mathrm{XZ}$ ' plane at the symmetry line $\mathrm{XC}$ '. There is a one to one correspondence on each side of the XC' line in the A' XZ' plane which maps the $H D_{X}$ to the $H D_{\perp}$ phase by exchanging rungs and diagonal. Consequently there is no crossing of the transition surface at any other place in the A'XZ' plane. The transition surface lies on the same side $\left(J_{X 1}>0\right)$ of the A'XC'L' triangle.

- On this side of the A'XZ' plane $\left(J_{X 1}>0\right)$, the ferromagnetic boundary is exactly known [Eq. (20)]. It has no singularity, which means that the transition surface between the two Haldane phases does not connect to it.

- From the two previous remarks, we conclude that the transition surface between the two Haldane phases abuts the ferromagnetic boundary along the curve $\mathrm{AC}^{\prime}$ in the $\mathrm{A}^{\prime} \mathrm{XZ}$ ' plane $\left(J_{X 1}=0\right)$ 


\section{The $[+,+,-]$ sector}

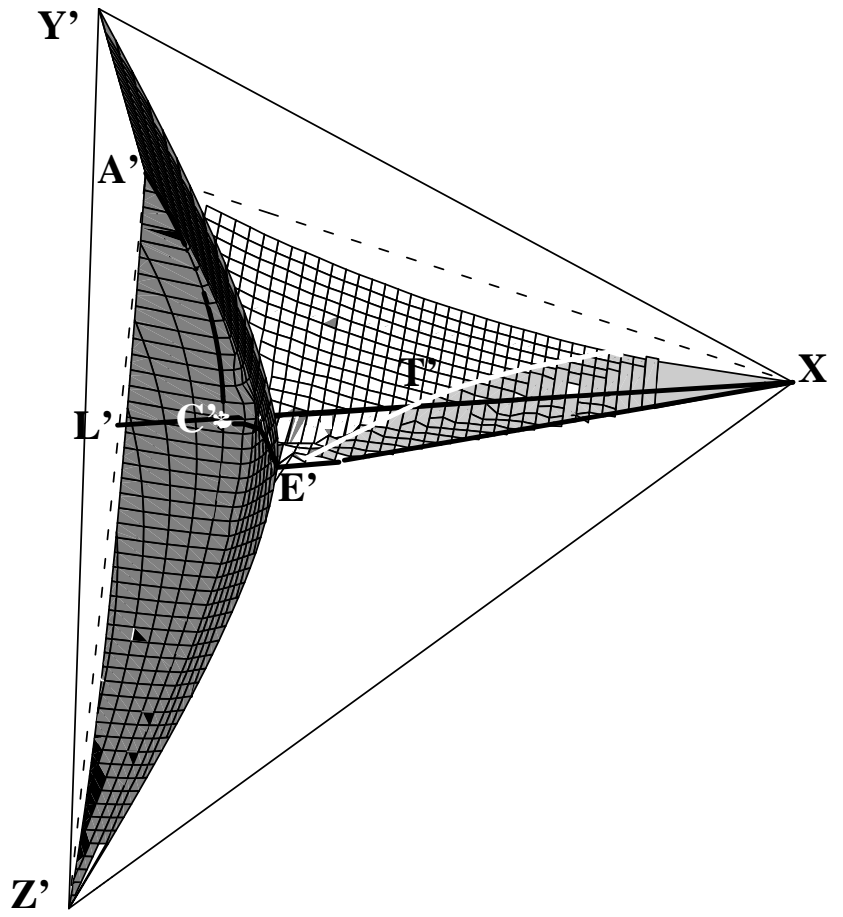

FIG. 23. The 3D phase diagram in the $[+,-,-]$ sector. The cut through the boundary face XY'Z' is shown in Fig. 22 and the cut through the plane A'XZ' corresponding to the "Zig-Zag chain" model has already been studied in Fig. 6 (b). The dark gray surface corresponds to the boundary of the ferromagnetic phase seen from inside. For $J_{X 1}>0$, the ferromagnetic boundary is given exactly through Eq. (20). The other side $J_{X 1}<0$ is calculated through DMRG. The other surface represents the transition between the two Haldane phases $H D_{X}$ and $H D_{\perp}$. The clear part corresponds to a first-order transition with finite gap and string-order parameters. The dark part is critical with vanishing gap and order parameter. The tricritical white line represents the boundary of the critical part of the surface. This surface abuts the ferromagnetic boundary along the curve A'C'E', which is represented from inside the ferromagnetic boundary as a heavy line. The part A'C' of this curve lies in the A'XZ' plane $\left(J_{\perp}=0\right)$ and coincides with the DKO line. It is a triple line.

The other part C'E' of this curve is clearly out of the A'XZ' plane. The meeting of this curve with the tricritical line is numerically found very close to E'. We have not been able however to prove that it coincides exactly with E'. We conclude that at least part of the curve C'E' is a triple line.
No phase transition has been found in this sector. The ground state is everywhere the $H D_{\perp}$ phase.

\section{The $[-,+,-]$ sector}

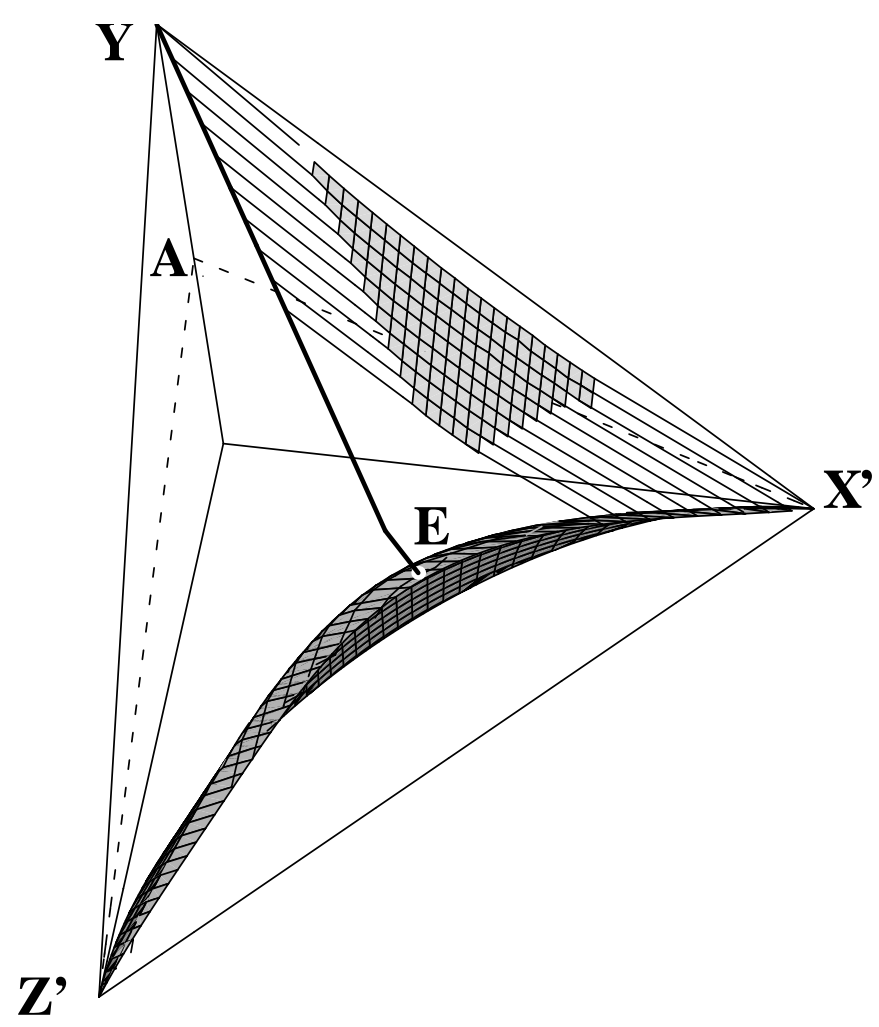

FIG. 24. The 3D phase diagram in the $[-,+,-]$ sector. The boundary face YX'Z' is obtained from the symmetrical diagram represented in Fig. 22 by changing $\mathrm{X}$ to $\mathrm{Y}$ and $\mathrm{Y}^{\prime}$ to $\mathrm{X}^{\prime}$. The dark surface represents the boundary of the ferromagnetic phase. Its intersection with the $\left(J_{X 2}=0\right) \mathrm{AX}^{\prime} \mathrm{Z}^{\prime}$ plane corresponds to the DKO exactly-soluble model. The $\left(J_{X 2}>0\right)$ part of this surface is known exactly [Eq. (20)]. The other part is calculated through DMRG. A part of the critical surface (clear-gray grid) corresponding to a second order transition between the two Haldane phases phases $H D_{X}$ and $H D_{\perp}$ has been determined through DMRG. It is very close to the boundary face X'YZ' but cuts it only on YE. A rough extrapolation of this surface (array of thin lines) is also shown; it indicates that the intersection of the critical surface with the ferromagnetic boundary is close to the X'YZ' face 
The three-dimensional phase diagram of the last $[-,+,-]$ sector is shown in Fig. 24. The ferromagnetic boundary (dark surface) is obtained in the same way as in other sectors: the part corresponding to $\left(J_{X 2}<0\right)$ is known exactly from Eq. (20). The complementary part is calculated through DMRG.

The cut by the X'YZ' face is obtained from Fig. 22 (XY'Z' face) through the symmetry which exchanges parallel and diagonal interactions. The transition surface between the two Haldane phases $H D_{X}$ and $H D_{\perp}$ lies everywhere close to this boundary plane and cuts it on YE.

Only a part of this surface (clear gray grid in Fig. 24) has been obtained, using our DMRG scheme, with reasonable accuracy. It is critical with vanishing gap and string order. The array of thin lines in Fig. 24 is a rough extrapolation. It indicates that the intersection of this surface with the ferromagnetic boundary lies also close to the boundary face X'YZ'.

\section{CONCLUSION}

We have presented an overall view on the threedimensional phase diagram of the frustrated two-leg ladder model. This model contains, as particular cases, the spin-1 Heisenberg chain with first and second nearestneighbor interactions, the spin- $\frac{1}{2}$ "Zig-Zag chain" with dimerization and frustration, the usual ladder and a variety of previously studied ladder models that we have briefly reviewed. We have proven that only three different phases occur: the ferromagnetic phase and two gapped Haldane phases with different symmetry and different long-range topological order. In a three dimensional phase diagram, these three phases are separated through transition surfaces. Some parts of the transition surface separating the two gapped Haldane phases are critical with vanishing gap and string-order parameters; the complementary parts corresponds to a first order transition with finite gap and two degenerate $S=0$ ground-states. In the two dimensional plane cuts of the three-dimensional phase-diagram which correspond to the "Zig-Zag chain model", there is a one to one mapping of these two Haldane phases through a simple symmetry which exchanges rung and diagonal interactions. Consequently, although the topology of these two Haldane phases differ, the critical behavior concerning their thermodynamic properties near the critical surface are the same and they belong to the same class of universality. Part of the boundary of the transition surface between these two Haldane phases with the ferromagnetic phase corresponds to the exactly-soluble DKO model. We expect that this result will suscitate further theoretical work on this model, concerning in particular remarkable points like $\Gamma$ and $C^{\prime}$ on the DKO line (see Figs. 18 and 23).

Most calculations were performed on the Cray T3E from CEA-Grenoble. Numerical data concerning the transition surfaces are available upon request $\$$

* Permanent address: Yerevan Physics Institute, Alikhanian br. 2, Yerevan Armenia.

$\dagger$ Permanent address: Michigan State University, Physics Department, East Lansing, 48824 MI USA.

¥ To whom correspondence should be addressed. Electronic address: roger@drecam.saclay.cea.fr

${ }^{1}$ E. Dagotto and T. M. Rice, Science 271, 618 (1996).

${ }^{2}$ H. Bethe, Z. Phys. 71, 205 (1931).

${ }^{3}$ F. D. M. Haldane, Phys. Rev. Lett. 50, 1153 (1983).

${ }^{4}$ S. R. White, Phys. Rev. B, 53, 52 (1996).

${ }^{5}$ Xiaoqun Wang, in Density-Matrix Renormalization, Lecture Notes in Physics, edited by I. Peschel, X. Wang, M. Kaulke and K. Hallberg, Springer (1999); Xiaoqun Wang, cond-mat/9803290 (unpublished).

6 S. R. White, Phys. Rev. Lett. 69, 2863 (1992); Phys. Rev. B 4810345 (1993).

${ }^{7}$ I. Affleck, T. Kennedy, E. H. Lieb, and H. Tasaki, Phys. Rev. Lett. 59, 799 (1987).

${ }^{8}$ P. W. Anderson, Mater. Res. Bull. 8, 153 (1973).

${ }^{9}$ K. Rommelse and M. den Nijs, Phys. Rev. Lett. 59, 2578 (1987).

${ }^{10}$ E. H. Kim, G. Fáth, J. Sólyom and D.J. Scalapino, condmat/9910023.

${ }^{11}$ A. Kolezhuk, R. Roth and U. Schollwöck, Phys. Rev. Lett. 77, 5142 (1996).

12 S. R. White and D. A. Huse, Phys. Rev. B 483844 (1993).

${ }^{13}$ C. K. Majumdar and D. K. Ghosh, J. Math. Phys. 10, 1388 (1969).

${ }^{14}$ B. S. Shastry and B. Sutherland, Phys. Rev. Lett. 47, 964 (1981).

${ }^{15}$ R. Chitra, Swapan Pati, H. R. Krishnamurthy, Diptiman Sen and S. Ramasesha, Phys. Rev. B 52, 6581 (1985).

${ }^{16}$ S. Brehmer, H.-J. Mikeska and U. Neugebauer, J. Phys. Cond. Mat. 8, 7161 (1996).

${ }^{17}$ V. J. Emery and C. Noguera, Phys. Rev. Lett. 60, 631 (1988). G. Castilla, S. Chakravarty and V. J. Emery, ibid. 75, 1823 (1995).

18 S. Eggert, Phys. Rev. B 54 R9612 (1999).

19 D. V. Dmitriev, V. Ya Krivnov, and A. A. Ovchinnikov, Phys. Rev. B 56, 5985 (1997); cond-mat/9911394 (1999).

${ }^{20}$ In Ref. 10, another view of "universality" is adopted, in which two phases which differ topologically are said to belong to different universality classes, even when they correspond through a simple reflection symmetry.

${ }^{21}$ A. K. Kohlezhuk and H.-J Mikeska, Int. J. Mod. Phys. B 12, 2325 (1998).

22 J. Sólyom and J. Timonen, Phys. Rev. B 34, 487 (1986); and references therein.

${ }^{23}$ M.A. Martin-Delgado, R. Shankar, and G. Sierra, Phys. Rev. Lett. 77, 3443 (1996).

24 M.A. Martin-Delgado, J. Dukelsky and G. Sierra, Phys. Lett. A 250, 430 (1998). 